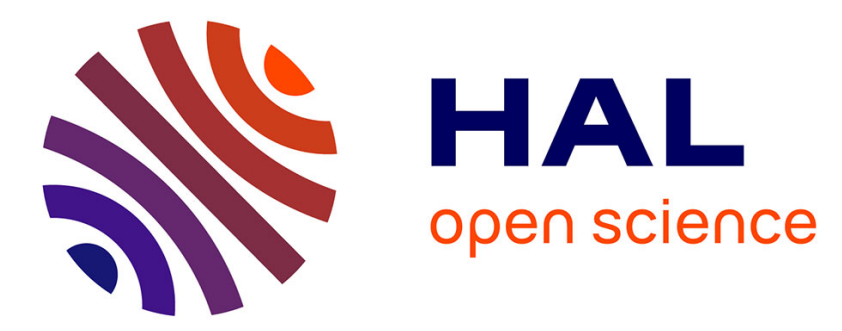

\title{
Probabilistic Tractography Using Q-Ball Imaging and Particle Filtering: Application to Adult and in-utero Fetal Brain Studies
}

Julien Pontabry, François Rousseau, Estanislao Oubel, Colin Studholme, Mériam Koob, Jean-Louis Dietemann

\section{To cite this version:}

Julien Pontabry, François Rousseau, Estanislao Oubel, Colin Studholme, Mériam Koob, et al.. Probabilistic Tractography Using Q-Ball Imaging and Particle Filtering: Application to Adult and in-utero Fetal Brain Studies. Medical Image Analysis, 2012, 17, pp.297-310. 10.1016/j.media.2012.11.004 . hal-00873625

\section{HAL Id: hal-00873625 \\ https://hal.science/hal-00873625}

Submitted on 16 Oct 2013

HAL is a multi-disciplinary open access archive for the deposit and dissemination of scientific research documents, whether they are published or not. The documents may come from teaching and research institutions in France or abroad, or from public or private research centers.
L'archive ouverte pluridisciplinaire $\mathbf{H A L}$, est destinée au dépôt et à la diffusion de documents scientifiques de niveau recherche, publiés ou non, émanant des établissements d'enseignement et de recherche français ou étrangers, des laboratoires publics ou privés. 


\title{
Probabilistic Tractography Using Q-Ball Imaging and Particle Filtering: Application to Adult and in-utero Fetal Brain Studies.
}

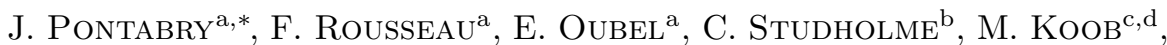 \\ J.-L. DiETEMAnN ${ }^{\mathrm{c}, \mathrm{d}}$ \\ ${ }^{a}$ LSIIT, UMR 7005 CNRS-Université de Strasbourg, France \\ ${ }^{b}$ Biomedical Image Computing Group, Departments of Pediatrics, Bioengineering and \\ Radiology, University of Washington, Seattle, USA \\ ${ }^{c}$ LINC, UMR 7237 CNRS-Université de Strasbourg, France \\ ${ }^{d}$ Service de Radiopédiatrie, Hôpital de Hautepierre, Strasbourg, France
}

\begin{abstract}
By assuming that orientation information of brain white matter fibers can be inferred from Diffusion-Weighted Magnetic Resonance Imaging (DW-MRI) measurements, tractography algorithms provide an estimation of the brain connectivity in-vivo. The two key ingredients of tractography are the diffusion model (tensor, high-order tensor, Q-ball, etc.) and the means to deal with uncertainty during the tracking process (deterministic vs probabilistic mathematical framework). In this paper, we investigate the use of an analytical Q-ball model for the diffusion data within a well-formalized particle filtering framework. The proposed method is validated and compared to other tracking algorithms on the MICCAI'09 contest Fiber Cup phantom. Tractographies of in-vivo adult and fetal brain Diffusion-Weighted Images (DWI) are also shown to illustrate the robustness of the algorithm.
\end{abstract}

Keywords: MRI, Tractography, Diffusion, MCMC, Particle filter

\footnotetext{
* Corresponding author

Email addresses: pontabry@unistra.fr (J. PONTABRY), rousseau@unistra.fr (F. Rousseau)
} 


\section{Introduction}

In the last decade, Magnetic Resonance Imaging (MRI) has become a popular and powerful tool for medical imaging and brain understanding. In particular, Diffusion-Weighted MRI is a non-invasive imaging system, which gives information on water diffusion in human brain. These indirect observations of the white matter geometry in vivo (Basser et al., 1994) allow nerve fiber reconstruction by using tractography algorithms. The two key points of a tractography algorithm are the diffusion model (Lenglet et al., 2009) and the mathematical framework describing the tracking process.

Water diffusion is historically modeled by diffusion tensors (Basser et al., 1994). This model is particularly suited to homogeneous regions of tissue fiber orientation, such as the corpus callosum. However, complex fiber architectures have been detected in approximately a third of voxels of the brain (Behrens et al., 2007) and the second-order tensor model does not handle such fiber bundle geometry well. A way to deal with these issues is to use High Angular Resolution Diffusion Imaging (HARDI) with appropriate diffusion models such as high-order tensors (HOT) (Liu et al., 2004, Özarslan and Mareci, 2003) or Q-ball (Tuch, 2004). The first is a generalization of the diffusion tensor and the Bloch-Torrey equation whereas Q-ball is a projection on the Q-space by a Funk-Radon transform. Q-Ball Imaging (QBI) has benefits over other HARDI reconstruction approaches by being linear and easy to compute (Tuch, 2004). In the last few years, spherical deconvolution (Barnett, 2009, Descoteaux et al., 2009, Tournier et al., 2007) and normalization (Aganj et al., 2010, Tristan-Vega et al., 2009) techniques have been developed to improve the accuracy Q-Ball modeling and its robustness to noise. In addition, the use of orientation diffusion functions $(\mathrm{ODF})$ in Q-Ball provides a straightforward density probability of the orientation of diffusion displacement.

The second key point in tractography algorithms concerns tracking process modeling. Tractography methods can be categorized using the two following features: the way they use DW-MRI data (local vs global modeling) and the 
underlying mathematical framework (deterministic vs probabilistic approaches) (see Lazar (2010) for a recent review of tractography methods).

The first proposed tractography methods (Basser et al., 2000, Mori et al., 1999) were local and deterministic. Starting from a seeding point, the fiber trajectory is computed in a step-wise fashion based on local measurement (such as the principal direction of the second order tensor). Stopping criteria are usually a brain mask (the trajectory exits the brain mask), low Fractional Anisotropy (FA) values (such as measured in CSF) or maximum curvature (assuming some smoothness of the fibers). These local deterministic approaches can be affected by the accumulation of errors and influenced by local irregularities in the diffusion data. A way to avoid such issues is to consider a filtering approach (Malcolm et al., 2010, Savadjiev et al., 2010). Another way to avoid such issues is to use a global framework for optimal trajectory estimation (Fillard et al., 2009, Jbabdi et al., 2007, Lifshits et al., 2009, Parker et al., 2002, Staempfli et al., 2006, Wu et al., 2009). Global optimization algorithms estimate fiber bundles using an energy-based framework. Global trajectories are obtained by optimizing global parameters related to the underlying diffusion data and possibly additional constraints such as fiber path smoothness. However, the estimation of the entire tractogram of the brain used to be often computationally expensive. Recent techniques, such as Reisert et al. (2011), require less computation time (few hours on a standard PC) to provide whole brain tractograms.

The fiber path estimation may be affected by local irregularities in diffusion data due to noise, partial volume effect or ambiguity induced by the diffusion model. A way to manage this uncertainty is to describe the tractography problem within a probabilistic framework. The uncertainty in fiber path estimation is then characterized by a set of possible propagation directions for a given voxel. Probabilistic methods generate multiple trajectories to provide a distribution of fiber bundles for a given seed point. The output of such algorithms is a probability map of connection between the given seed voxel and other voxels of the brain. Probabilistic streamline (Berman et al., 2008), probabilistic optimization-based (Jbabdi et al., 2007, Lifshits et al., 2009) and stochastic 
path sampling (Behrens et al., 2007, 2003, Bjornemo et al., 2002, Friman et al., 2006, Jeurissen et al., 2011, Lazar and Alexander, 2005, Parker and Alexander, 2003) algorithms can be discerned. In such a framework, a key point is the effectiveness of the sampling stage (Parker and Alexander, 2003, Zhang et al., 2009). In particular, as fiber paths can be modeled as Markov chains, Markov Chain Monte Carlo (MCMC) methods can be efficiently used for the sampling stage (Zhang et al., 2009).

Extending previous work (Zhang et al., 2009), we investigate in this study the use of a high order modeling, such as Q-ball within a particle filtering framework. The reliability of these recent models allows us to extract more information from data, such as the orientation distribution function (ODF) which provides a precise idea of the underlying fiber architecture at every voxel. Then, a nonlinear state-model is used for the tracking modeling and the probabilistic maps of fibers are estimated using a particle filtering algorithm. Therefore, the proposed algorithm depends only on spherical functions, such as ODF, and not on the diffusion model used. Experimental results on synthetic data, phantom data and real data, both adult and fetal, illustrate the robustness of the proposed algorithm.

\section{Theory}

The tractography challenge can be treated as a tracking process where the trajectory of the most likely fiber path is evaluated. MCMC methods are usually employed to solve this kind of problem. First, the fiber trajectory is modeled as a Markov Chain. Then, the posterior density of the fiber's trajectory is estimated using a Sequential Importance Sampling (SIS) framework (Doucet et al., 2000).

The tractography problem is formulated as a dynamic system. Using a state space model, prior probability defines the expected trajectory of a fiber and likelihood probability characterizes the uncertainty of its geometry. Considering a non-Gaussian state space model, prior and observation densities are non- 

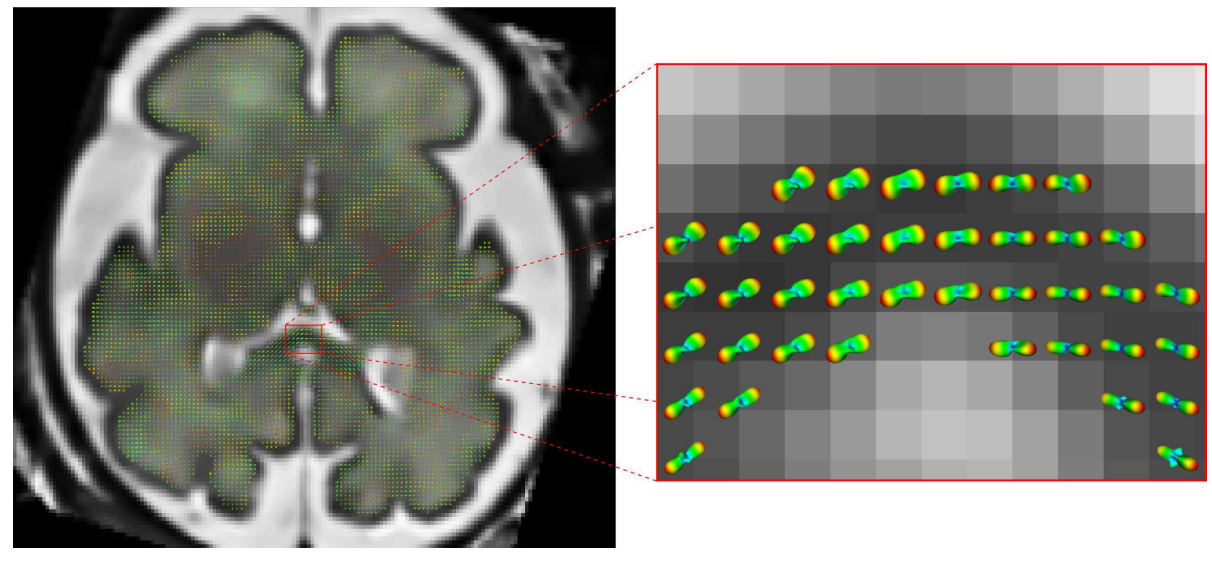

Figure 1: As observations, the algorithm use spherical functions describing the orientation probability of the diffusion at each location in the volume, i.e. the orientation of fiber bundles. In this work, Descoteaux et al. (2009) is used for diffusion modeling (see Appendix A). It can be noted that the presented model is sufficient for the data processed in this work, but the presented algorithm can use any recent model providing such spherical functions. On the illustration, a field of spherical function is plotted on an axial slice of a fetal brain (the model order is $l=4$ ).

Gaussian. The problem of white matter tractography is then a dynamic nonlinear system. Contrary to linear filtering methods such as Kalman filtering, particle filtering techniques are well suited to estimating the posterior density of non-linear systems.

\subsection{Observations}

The raw diffusion signal (i.e. DWI data) forms the observations of the process but it is not used directly. The algorithm need rather spherical functions $\psi_{x}$ of the orientation of the diffusion along fiber bundles at each location $x$ in the image volume (see figure 1). These functions model a spherical probability of the fiber configuration at $x$ and can be computed from the DWI data.

In this work, a spherical harmonics modeling of water diffusion in brain is used (Descoteaux et al., 2009). The two main advantages of this model are its accuracy (with respect to the second order tensor model) and its analytical form. The diffusion signal is decomposed into spherical harmonics and the fODF is 
then computed by applying the Funk-Radon theorem (see Appendix A and Descoteaux et al. (2009) for more details). The experiments in this work reveal that the chosen modeling of diffusion provides satisfactory brain tractograms on such data (low b-factor and moderate number of gradient directions). Nevertheless, other diffusion models could be considered (Aganj et al., 2010, Barnett, 2009, Tournier et al., 2007, Tristan-Vega et al., 2009). Since the algorithm only needs a spherical function modeling the distribution of the fibers configuration at any location in the image volume, any of these models can be used instead.

Considering the diffusion modeling (Descoteaux et al., 2009), the fODF is normalized between 0 and 1 . In our experiments, this diffusion modeling produces satisfactory results for order $l=4$. For higher order, spurious and negative peaks can be removed if needed by applying a low pass filter on it (as used in Tournier et al. (2007)). During the process, the maxima of the spherical functions have to be extracted. Several methods are available in the literature (Bloy and Verma, 2008, Jiao et al., 2011, Schultz and Seidel, 2008). In this work, a space discretization and an exhaustive search has been used. Due to noise, small peaks can appear on the spherical function. As used in Descoteaux et al. (2009), a threshold on the fODF magnitude has been implemented in order to avoid the extraction of such peaks (see Descoteaux et al. (2009) for details).

\subsection{Fiber trajectory model}

In a volume $\Omega \subset \mathbb{R}^{3}$, a fiber trajectory can be modeled as a sequence of $n$ displacement vectors $v_{k}$ with $k=1, \ldots, n$. From a given starting point $x_{0} \in \Omega$, each point of the path is defined recursively as

$$
x_{k+1}=x_{k}+\lambda v_{k}
$$

where $\lambda \in \mathbb{R}$ is the step size which is assumed to be constant in this work and $v_{k}$ is a unit vector at step $k$. Hence, in space state model, the trajectory $v_{0: k}$ can be built iteratively. The fiber trajectory model is depicted in figure 2. During the sequential construction of the solution, at each step, only local information of the diffusion is available. Therefore, fiber paths are assumed to have Markovian 


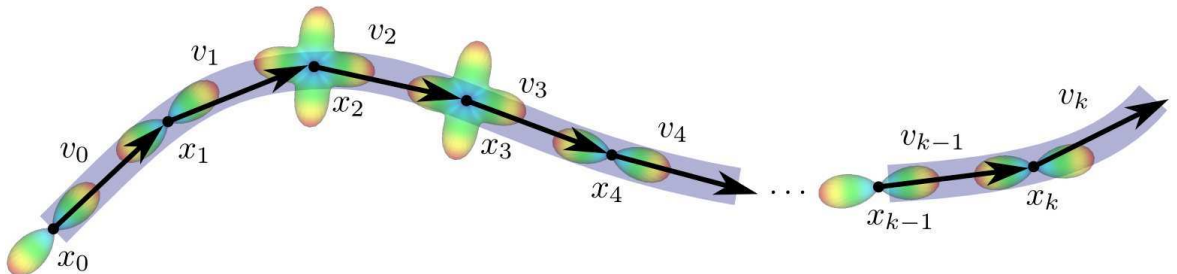

Figure 2: A fiber trajectory is modeled as a sequence of displacement vectors. The real fiber path is displayed in blue and the corresponding sequence of vectors is illustrated by black arrows and points. Diffusion data is shown at each point. Considering states $y_{t}=\left\{x_{t}, v_{t}\right\}$ for $t=0, \ldots, k$, the fiber path modeling forms a Markov chain since each state depends only on previous state and observations.

nature. As illustrated in figure 2, this means that each step depends only on previous step and on current observation data.

\subsection{Fiber tracking model}

Observations are DWI intensities in a volume $\Omega \in \mathbb{R}^{3}$ and the fiber trajectory model consists in a sequence of displacement vectors in $\Omega$. The displacement vectors are the parameters to be estimated. For this purpose, the Sequential Importance Sampling (SIS) algorithm is used (see algorithm 1).

\subsubsection{Sequential Importance Sampling (SIS)}

Let the DWI intensities be denoted as $\mathcal{U}$. Given a location $x \in \Omega$, the diffusion signal $\mathcal{S}_{x}$ and the fODF $\psi_{x}$ are constructed from intensities $\mathcal{U}_{x}$. In a dynamic system, state and observation process, respectively $y_{k}=\left\{x_{k}, v_{k}\right\}$ and $z_{k}=\left\{\mathcal{U}_{x_{k}}\right\}$, are defined as follow:

$$
\left\{\begin{array}{l}
y_{k}=\mathrm{f}_{k}\left(y_{k-1}, \gamma_{k}\right) \\
z_{k}=\mathrm{h}_{k}\left(y_{k}, \delta_{k}\right)
\end{array},\right.
$$

where no linear assumption is made on functions $\mathrm{f}_{k}$ and $\mathrm{h}_{k}$ and where $\gamma_{k}$ and $\delta_{k}$ are independent, eventually Gaussian, white noise. The dynamic system is entirely defined by three densities : initial, prior and likelihood (Doucet et al., 2000). 


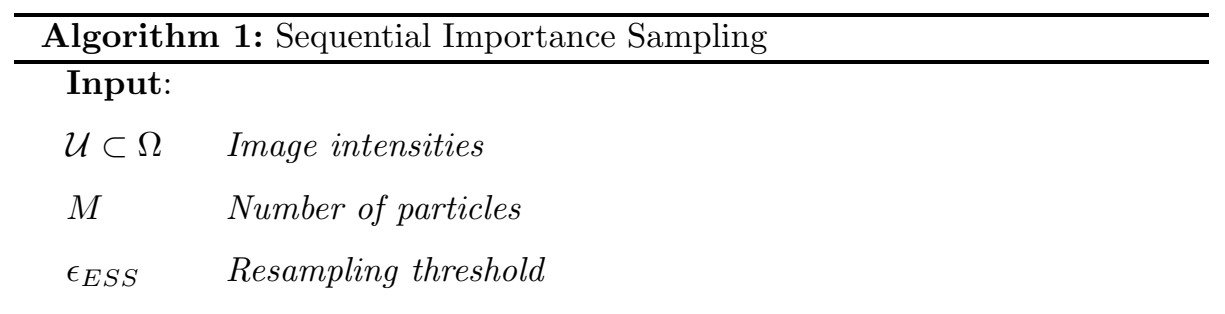

\section{Output:}

$\mathcal{C}=\left\{y_{0: k}^{(m)}, \tilde{w}_{0: k}^{(m)}\right\}_{m=1}^{M} \quad$ Particles' cloud

foreach particle in $\mathcal{C}$ do

$\mid \begin{aligned} & y_{0} \sim p\left(y_{0}\right) \\ & \tilde{w}_{0}=\frac{1}{M}\end{aligned}$

for $k=1$ to $K$ do

foreach particle in $\mathcal{C}$ do

$y_{k} \sim \pi\left(y_{k} \mid \Theta\left(y_{k-1}, z_{k}\right)\right)$

$w_{k}=w_{k-1} \frac{p\left(z_{k} \mid y_{k}\right) p\left(y_{k} \mid y_{k-1}\right)}{\pi\left(y_{k} \mid \Theta\left(y_{k-1}, z_{k}\right)\right)}$

$\tilde{w}_{k}=\frac{w_{k}}{\sum w_{j}}$

end

$N_{\text {ESS }}=\frac{1}{\sum\left(\tilde{w}_{j}\right)^{2}}$

if $N_{E S S}<\epsilon_{E S S}$ then

| resample all particles (last state) according to importance weights end

end 
Conditionally to step $k$, measures at same and next step are assumed to be independent:

$$
p\left(z_{k}, y_{k+1} \mid y_{k}\right)=p\left(z_{k} \mid y_{k}\right) p\left(y_{k+1} \mid y_{k}\right) .
$$

Due to the Markovian nature of fiber trajectory and independent condition given by (3), a fiber path is modeled as a Markov chain. Thus, dynamic system is only described by the following densities:

$$
\begin{gathered}
p\left(y_{0}\right), \\
p\left(y_{k} \mid y_{0: k-1}, z_{1: k-1}\right)=p\left(y_{k} \mid y_{k-1}\right), \\
p\left(z_{k} \mid y_{0: k}, z_{1: k-1}\right)=p\left(z_{k} \mid y_{k}\right) .
\end{gathered}
$$

Considering (3), (5) and (6) and applying Bayes theorem, we have an expression of the posterior density:

$$
\begin{aligned}
p\left(y_{0: k} \mid z_{1: k}\right) & =p\left(y_{0: k} \mid z_{1: k-1}, z_{k}\right) \\
& =\frac{p\left(y_{0: k}, z_{k} \mid z_{1: k-1}\right)}{p\left(z_{k} \mid z_{1: k-1}\right)} \\
& \propto p\left(z_{k} \mid y_{0: k}, z_{1: k-1}\right) p\left(y_{0: k} \mid z_{1: k-1}\right) \\
& \propto p\left(z_{k} \mid y_{k}\right) p\left(y_{k} \mid y_{0: k-1}, z_{1: k-1}\right) p\left(y_{0: k-1} \mid z_{1: k-1}\right) \\
& \propto p\left(z_{k} \mid y_{k}\right) p\left(y_{k} \mid y_{k-1}\right) p\left(y_{0: k-1} \mid z_{1: k-1}\right) .
\end{aligned}
$$

The principle of particle filtering is to sample sequentially a set of $M$ paths from the starting point $x_{0} \in \Omega$. This means that $M$ weighted particles are placed at point $x_{0}$ at step $k=0$ and are propagated as time progresses. Given the set of particles $\left\{y_{0: k}^{(m)}, w_{k}^{(m)}\right\}_{m=1}^{M}$ at step $k$, the propagation to the next step $k+1$ is performed following three stages: prediction, weighting and selection. In prediction stage, since the posterior $p\left(y_{0: k} \mid z_{1: k}\right)$ cannot be evaluated, the importance density $\pi\left(y_{0: k} \mid z_{1: k}\right)$ (which is an approximation of the posterior density) is used to simulate each of the vectors at each point in the fiber paths. Assuming causality of the importance density, i.e. for all $t \geq k, \pi\left(y_{0: k} \mid z_{1: t}\right)=\pi\left(y_{0: k} \mid z_{1: k}\right)$, a recursive formulation along the path can be chosen (Doucet et al., 2000), such that

$$
\pi\left(y_{0: k} \mid z_{1: k}\right)=\pi\left(x_{0}\right) \prod_{t=1}^{k} \pi\left(y_{t} \mid y_{0: t-1}, z_{1: t}\right)
$$


which leads to

$$
\pi\left(y_{0: k} \mid z_{1: k}\right)=\pi\left(y_{k} \mid y_{0: k-1}, z_{1: k}\right) \pi\left(y_{0: k-1} \mid z_{1: k-1}\right)
$$

Therefore, at step $k$, by considering the Markovian nature of fiber paths, the state $y_{k}$ of a path $y_{0: k-1}$ is sampled according to $\pi\left(y_{k} \mid y_{0: k-1}, z_{1: k}\right)$.

After the prediction stage, a weighting stage giving an estimate of the reliability of the approximation of the posterior density is performed. Using a ratio between the unknown posterior distribution and its approximation, a particle's weight is given by

$$
w_{k}^{(m)}=\frac{p\left(y_{0: k}^{(m)} \mid z_{1: k}\right)}{\pi\left(y_{0: k}^{(m)} \mid z_{1: k}\right)} .
$$

By inserting (7) and (9) in (10), we get a recursive definition of a particle's weight:

$$
\begin{aligned}
w_{k}^{(m)} & =\frac{p\left(y_{0: k}^{(m)} \mid z_{1: k}\right)}{\pi\left(y_{0: k}^{(m)} \mid z_{1: k}\right)} \\
& \propto \frac{p\left(z_{k} \mid v_{k}^{(m)}\right) p\left(y_{k}^{(m)} \mid y_{k-1}^{(m)}\right) p\left(y_{0: k-1}^{(m)} \mid z_{1: k-1}\right)}{\pi\left(y_{k}^{(m)} \mid y_{0: k-1}^{(m)}, z_{1: k}\right) \pi\left(y_{0: k-1}^{(m)} \mid z_{1: k-1}\right)} \\
& \propto w_{k-1}^{(m)} \frac{p\left(z_{k} \mid y_{k}^{(m)}\right) p\left(y_{k}^{(m)} \mid y_{k-1}^{(m)}\right)}{\pi\left(y_{k}^{(m)} \mid y_{0: k-1}^{(m)}, z_{1: k}\right)} .
\end{aligned}
$$

Here the weight $w_{k}^{(m)}$ at step $k$ is computed using the weight $w_{k-1}^{(m)}$ at step $k-1$, the prior density $p\left(y_{k}^{(m)} \mid y_{k-1}^{(m)}\right)$, the likelihood density $p\left(z_{k} \mid y_{k}^{(m)}\right)$ and the importance density $\pi\left(y_{k}^{(m)} \mid y_{0: k-1}^{(m)}, z_{1: k}\right)$. A normalization step is then applied:

$$
\tilde{w}_{k}^{(m)}=\frac{w_{k}^{(m)}}{\sum_{n=1}^{M} w_{k}^{(n)}} .
$$

Choosing an importance distribution of the form (9) leads to an increasing variance of the weights as the time progresses (Kong et al., 1994). As a result, early in the estimation process, a significant fraction of the weights may fall rapidly. So, the purpose of the final stage selection is to avoid this degeneracy. We first measure degeneracy of the cloud of paths using an estimate of the effective sample size (ESS) (Kong et al., 1994, Liu, 1996):

$$
\widehat{N_{\mathrm{ESS}}}=\frac{1}{\sum_{m=1}^{M}\left(\tilde{w}_{k}^{(n)}\right)^{2}} .
$$


When $N_{\text {ESS }}$ decreases below a fixed threshold $\varepsilon_{\text {ESS }}$, a resampling procedure is applied in order to eliminate particles with low weight (Doucet et al., 2000).

\subsubsection{Densities}

To help illustrate the form of the distributions and understand the components of the Bayesian framework, figure 4 shows the configurations of the distributions for a simple example of crossing fibers.

Prior density. As in (Zhang et al., 2009), the von Mises-Fisher (vMF) distribution has been selected as the prior because it is a parametric distribution for directional data. Another common choice in the literature is the Watson distribution (see for instance Malcolm et al. (2010)). On the ( $d-1)$-dimensional sphere, vMF distribution of unit vector $x \in \mathbb{R}^{d}$ is given by

$$
\mathrm{f}_{d}(x \mid \mu, \kappa)=\mathrm{C}_{d}(\kappa) \mathrm{e}^{\kappa \mu^{\mathrm{T}} x},
$$

where $\kappa \geq 0,\|\mu\|=1$ and $\mathrm{C}_{d}(\kappa)$ is a normalization constant defined as

$$
\mathrm{C}_{d}(\kappa)=\frac{\kappa^{d / 2-1}}{(2 \pi)^{d / 2} \mathrm{I}_{d / 2-1}(\kappa)}
$$

where $I_{d / 2-1}$ denotes the modified Bessel function of the first kind and order $\frac{d}{2}-1$. This distribution is parametrized by $\mu$ and $\kappa$, respectively called mean direction and concentration. The greater the value of $\kappa$, the stronger is the concentration of the distribution around mean direction $\mu$. The distribution is uniform over the sphere if $\kappa=0$ and when $\kappa \rightarrow \infty$, the distribution is focused on a point of the sphere defined by $\mu$.

In this work, directions are defined on the 2-dimensional unit sphere in $\mathbb{R}^{3}$. Thus, the prior is defined by a vMF distribution in dimension $d=3$ and a concentration parameter $\kappa$ :

$$
p\left(y_{k} \mid y_{k-1}\right)=\mathrm{f}_{3}\left(u_{k} \mid u_{k-1}, \kappa\right),
$$

where $u_{k}$ and $u_{k-1}$ are spherical coordinates versions of respectively vectors $v_{k}$ and $v_{k-1}$. The value of concentration parameter $\kappa$ is a smoothness constraint of the fiber path. It can be set manually as a general parameter of the algorithm. 
Importance density. The iterative solution estimation relies on the approximation of the posterior, i.e. on the importance density. At each step, knowing a path's vector sequence and its observation information, next direction added to the path is sampled according to the importance density. The optimal importance density is $p\left(y_{k} \mid y_{k-1}, z_{1: k}\right)$, because conditionally upon $y_{k}$ and $y_{1: k}$, the variance of the importance weights $w_{k}$ is then minimal (Doucet et al., 2000). Since it is difficult to sample from $p\left(y_{k} \mid y_{k-1}, z_{1: k}\right)$, a usual choice is to use the same distribution as prior for importance. Nevertheless, this approach, called bootstrap filter or condensation algorithm, may not be efficient enough : since no observation information is used, the particles generated using the prior are often outliers of the true posterior distribution.

As importance function, we choose a local parametrization $\pi\left(y_{k} \mid \theta\left(y_{k-1}, z_{k}\right)\right)$, where $\theta\left(y_{k-1}, z_{k}\right)$ is a finite dimensional parameter determined by $y_{k-1}$ and $z_{k}$ (Doucet et al., 2000). The ODF functions model water diffusion in the brain and give an estimation of the underlying fiber architecture. Each ODF's maxima should indicate fibers' directions. Let $\Lambda_{k}$ be a set of directions in the solid angle defined by a threshold angle $\theta$ around the previous direction $v_{k-1}$, where $\psi_{x_{k}}$ is locally maximum. Then, importance density is defined in dimension $d=3$ as a vMF mixture:

$$
\pi\left(y_{k} \mid \theta\left(y_{k-1}, z_{k}\right)\right)=\left\{\begin{array}{cl}
\sum_{\mu \in \Lambda_{k}} \omega_{\mu} \mathrm{f}_{3}\left(u_{k} \mid \mu, \kappa_{\mu}\right) & \text { if } \Lambda_{k} \neq \emptyset \\
\mathrm{f}_{3}\left(u_{k} \mid u_{k-1}, \kappa\right) & \text { else }
\end{array}\right.
$$

where $u_{k}$ is the unit vector in spherical coordinates corresponding to the unit vector $v_{k}$ in Cartesian coordinates, $\kappa_{\mu}$ is the concentration depending on observations, $\kappa$ is the concentration parameter and $\omega_{\mu}$ are mixture proportions such that $0 \leq \omega_{\mu} \leq 1$ and $\sum_{\mu \in \Lambda_{k}} \omega_{\mu}=1$. Each $\omega_{\mu}$ is proportional to the ODF value in direction $\mu$ :

$$
\omega_{\mu}=\left\{\begin{array}{cl}
\frac{\Psi_{x_{k}}(\mu)}{\sum_{\nu \in \Lambda_{k}} \Psi_{x_{k}}(\nu)} & \text { if } \widehat{u_{k-1} \mu} \in[-\Theta, \Theta] \\
0 & \text { else }
\end{array} .\right.
$$

The use of a vMF mixture leads to an efficient sampling procedure, in computation time, of the importance distribution (Ulrich, 1984). 


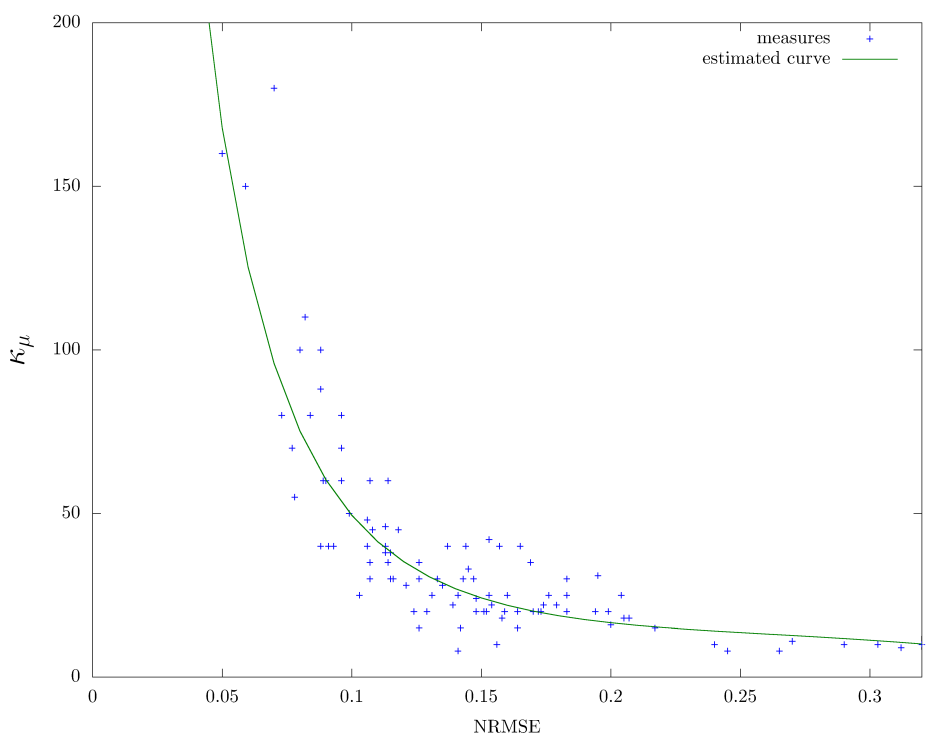

Figure 3: Fitting concentration parameter $\kappa_{\mu}$ as a function of NRMSE in direction $\mu$.

The more fitted are intensities $\mathcal{U}_{x_{k}}$ to model $S_{x_{k}}$, the more focused in direction $\mu$ should be the importance distribution. Thus, concentration parameter $\kappa_{\mu}$ can be set according to the uncertainty of the model in the direction $\mu$. The Normalized Root Mean Square Error (NRMSE) of the model gives a measure of fitting between the intensities and the model. Let $\xi_{\mu}$ be the NRMSE restricted to the solid angle defined by a threshold angle $\theta$ around the mean direction $\mu$. The parameter $\kappa_{\mu}$ can be empirically computed as a function of $\xi_{\mu}$. The figure 3 indicates that the relationship between concentration parameter $\kappa_{\mu}$ and $\xi_{\mu}$ is well described by an exponential function:

$$
\kappa_{\mu}=\alpha \exp \left(\beta \xi_{\mu}+\gamma \xi_{\mu}^{2}\right)
$$

where $\alpha, \beta$ and $\gamma$ are estimated from the fitting in figure 3 .

Likelihood density. Partially due to noise, there is uncertainty in MRI diffusion information. The observation density is supposed to be a measure of this uncertainty. In term of probabilities, likelihood defines a measure of how observations match the current model. We model the likelihood density as a distance error 
between measured observations and observations matching perfectly the current state model. At step $k, u_{k}$ is the direction sampled according to the importance and $\mu$ is assumed to be the mean vector of the sampled vMF distribution (in order to get a direction $u_{k}$ ). According to section Appendix A.2, the diffusion signal at position $x_{k}$ is described by $S_{x_{k}}$. Let $\tilde{S}_{x_{k}}$ be the diffusion signal at position $x_{k}$ if the sampled direction $u_{k}$ was exactly the mean direction $\mu$, i.e. $\mu=u_{k}$. Then, $\tilde{S}_{x_{k}}$ is determined from $S_{x_{k}}$ by a rotation of angle $\widehat{\mu u_{k}}$ in three dimensional space:

$$
\tilde{S}_{x_{k}}=\mathcal{R o t}\left(\widehat{\mu u_{k}}, S_{x_{k}}\right),
$$

where $\widehat{\mu u_{k}}$ denotes the angle between $\mu$ and $u_{k}$.

Let consider $\tilde{S}_{x_{k}}$ as the diffusion signal and the MRI intensities $\mathcal{U}_{x_{k}}$ a noisy version of it, i.e. $\mathcal{U}_{x_{k}}=\tilde{S}_{x_{k}}+\epsilon$. Noise in MRI images can be described for moderate-large signal-to-noise ratio (SNR $\geq 4 \mathrm{~dB}$ ) by a normal distribution (Nowak, 1999), i.e. $\epsilon=\mathcal{U}_{x_{k}}-\tilde{S}_{x_{k}} \sim \mathcal{N}\left(0, \Sigma^{2}\right)$. The standard deviation of diffusion weighted image in each gradient directions $\sigma_{i}$ is estimated by least square estimation and pseudo-residuals (Gasser et al., 1986). Assuming that the error distributions in each gradient direction $g_{i}$ are independent, the likelihood is given by:

$$
\begin{aligned}
p\left(z_{k} \mid v_{k}\right) & =\prod_{i=1}^{N} \mathcal{N}\left(\mathcal{U}_{x_{k}}\left(g_{i}\right)-\tilde{S}_{x_{k}}\left(g_{i}\right) \mid 0, \sigma_{i}^{2}\right) \\
& =\prod_{i=1}^{N} \frac{1}{\sigma_{i} \sqrt{2 \pi}} \mathrm{e}^{-\frac{1}{2}\left(\frac{\mathcal{U}_{x_{k}}\left(g_{i}\right)-\tilde{S}_{x_{k}}\left(g_{i}\right)}{\sigma_{i}}\right)^{2}} .
\end{aligned}
$$

If there is no mean direction $\mu$ in the solid angle defined by an threshold angle $\theta$ around $v_{k-1}$, i.e. $\Lambda_{k}=\emptyset$, likelihood is set to 1 . Thus, particles are weighted considering only prior and importance densities.

\subsection{Algorithm's behaviour}

The particle filtering algorithm propagates for each seed a cloud of particles representing the density probability of the fiber path passing through the seed voxel. When some particles reach a multi-directionnal point, such as crossing, the cloud splits in any possible direction which fullfill the angle condition and 


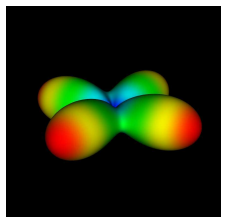

(a) fODF

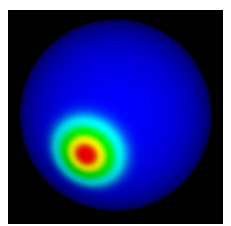

(b) Prior density

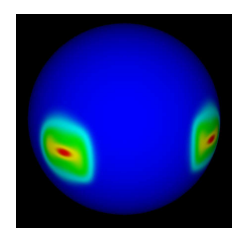

(c) Likelihood density

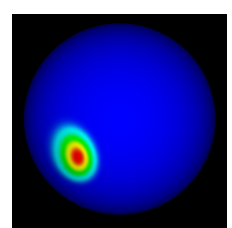

(d) Posterior density

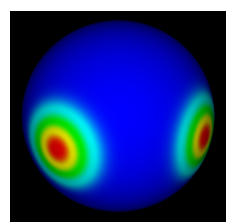

(e) Importance density

Figure 4: Likelihood, prior and posterior densities are displayed above for 2 crossing fibers configuration. The corresponding importance density is shown too. Starting from the fODF, the Bayesian framework of the particle filtering method is illustrated here. Posterior density is defined as the product of prior and likelihood density. The final weighting function of the particles includes the fraction of the posterior density on the importance density.

the parameters of the probabilistic model with respect to the previous direction. The sampling proportions in each direction depends on the probability of each direction defined by the densities used in the algorithm (see section 2.3.2). The output connectivity map should reflect the integrated motion of the particles. Then, the fiber path is estimated by computing the MAP of the posterior density.

\subsection{Connectivity map and fiber bundle estimation}

The SIS algorithm provides as output a particles cloud which approximates the posterior density. This cloud is not the expected output data structure, such as connectivity map and fiber pathway. Both representation are important: the connectivity map represents the uncertainty of the process whereas the fiber tract represents the estimated optimal path. The probability map of connections from the seed point is computed as the frequency of particles passing through each voxel. It could have been generated using the importance weights, however, in our experiments, the two methods lead to very similar maps.

The fiber trajectory estimate can be approximated by the MAP of the posterior density. Usually, particle's trajectory with the higher importance weight is used as MAP estimate (Zhang et al., 2009). Nevertheless, this choice induces irregularities of trajectory due to both the resampling stage and the probabilis- 
tic simulations. A better approach is to search the optimal pathway in particles cloud. This solution is at least equal to the particle's trajectory with the best importance weight (Godsill et al., 2001). This process is detailed in algorithm 2.

\subsection{Implementation considerations}

The discrete nature of DWI requires algorithms working in a discrete space. When a particle fall between two voxels of the image domain, the properties of this particular location, such as the diffusion signal, have to be interpolated. This can implies several issues: interpolation of the data and its impact on the tractography algorithm. The chosen model provide a solution to the first issue. Indeed, this model is analytical and linear, allowing to interpolate directly the spherical harmonics coefficients. During the experiments, the data have been interpolated using B-Spline of order 1 (equivalent to trilinear interpolation). The second issue raises essential problems on boundaries, when the diffusion signal changes drastically (e.g. the boundary between the white matter and the cerebrospinal fluid). The interpolated spherical functions on the boundaries may not be correct. In this work, a mask has been used to prevent the particles going out the white matter, which is a common strategy (Descoteaux et al., 2009, Savadjiev et al., 2010).

\section{Experimental results}

For all the experiments presented in this article, the stopping criterion of the tractography algorithm is the exit of all particles of a predefined mask and the chosen model is used at order $l=4$. Other parameters of the modeling are set as advised in Descoteaux et al. (2009).

\subsection{Synthetic data}

For validation purposes, a synthetical slice of size $16 \times 16 \mathrm{~mm}$ representing two crossing fiber bundles has been used (figure 5). The dataset has been generated using Matlab code of Barmpoutis et al. (2009). 


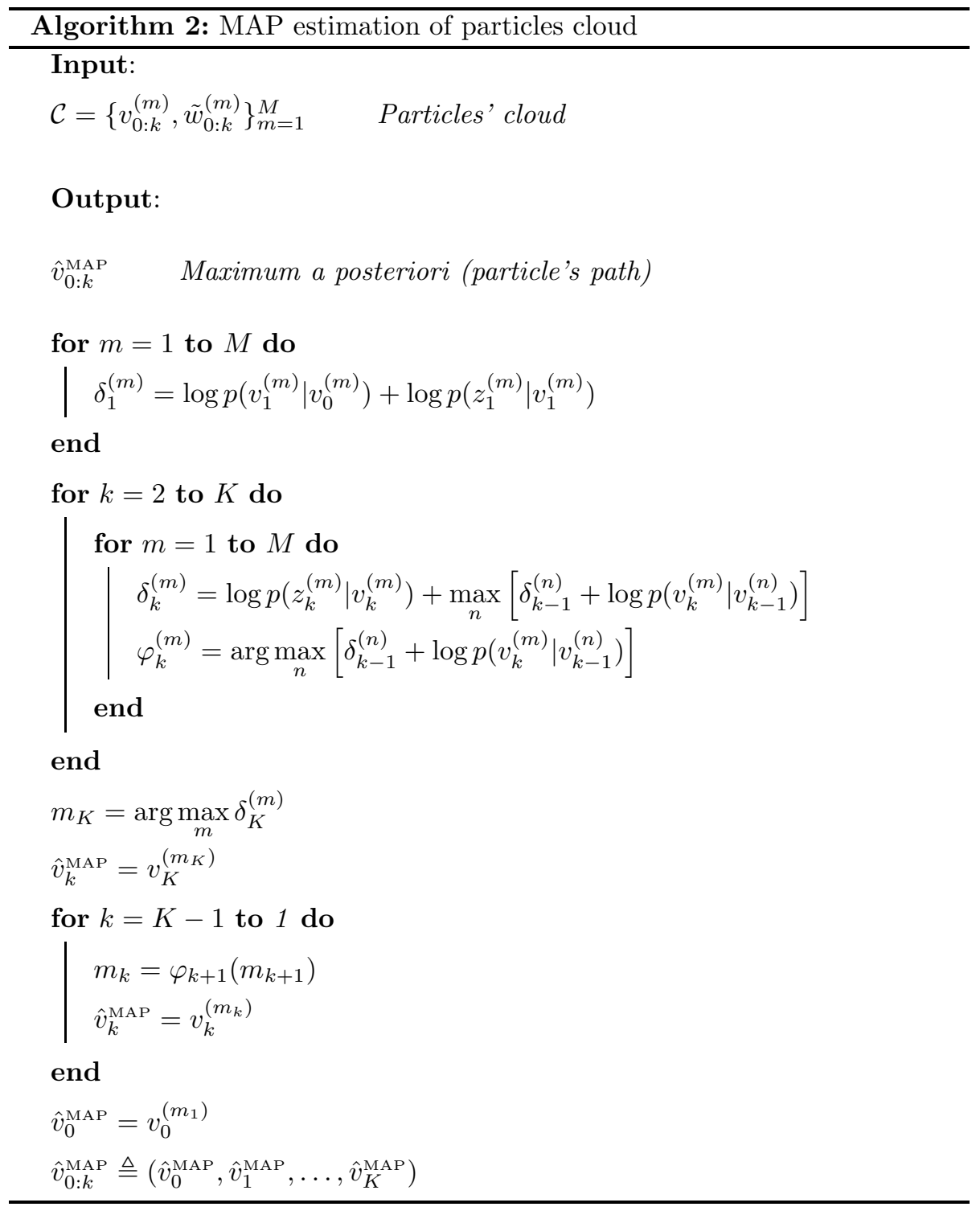




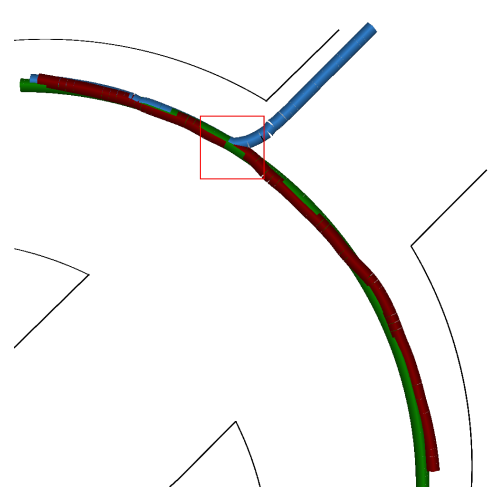

(a) Synthetic dataset

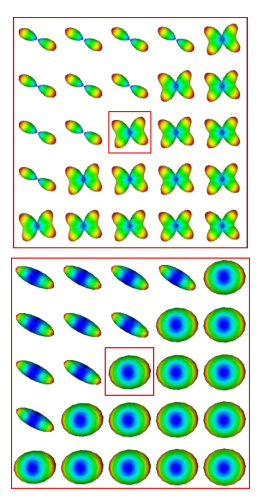

(b) Tensor and fODF fields
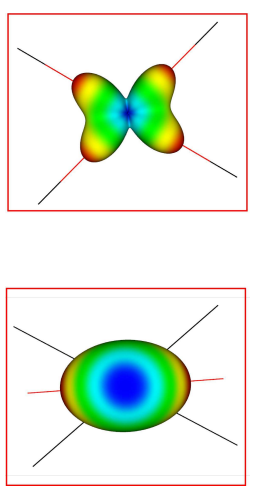

(c) Tensor and fODF

Figure 5: A tractography of a synthetic dataset is shown in 5(a). The ground truth, the result of Zhang's algorithm and the proposed algorithm are respectively displayed in green, blue and red. Details of the fODF and tensor fields located in the red square in the dataset 5(a) are exposed in 5(b). In 5(c), both fODF and tensor located in the red squares in 5(b) are depicted. The directions estimated by the model and the true directions are illustrated respectively by red and black lines.

Figure 5(a) shows the results obtained with the proposed method compared to our implementation of the tensor-based approach of Zhang et al. (2009). The proposed algorithm (in red) is able to track the ground truth (in green) whereas the tensor-based algorithm (in blue) fails on the crossing configuration. As shown by details of tensor and fODF fields in figure 5 , the tensor model does not capture the local diffusion directions and may mislead the tracking algorithm.

\subsection{Fiber Cup phantom}

The Fiber Cup is a tractography contest proposed at the MICCAI conference help in London in $2009^{1}$. By containing several crossing, kissing, splitting and bending fiber configurations, this phantom (Poupon et al., 2008) is

\footnotetext{
${ }^{1}$ The website http://www.lnao.fr/spip.php?rubrique79 provides details and results about this contest. The phantom used to compare tractographies and the comparison program are also available on this website.
} 
Table 1: Quantitative results of the proposed algorithm on the Fiber Cup phantom compared to the contest winner's quantitative results.

\begin{tabular}{c||c|c|c||c|c|c}
\hline \multicolumn{1}{c||}{ Fibers } & \multicolumn{3}{c||}{ Proposed algorithm } & \multicolumn{3}{c}{ Reisert et al. $(2011)$} \\
& L2 & tan & curv & L2 & tan & curv \\
\hline \hline F1 & 2.39 & 14.78 & 0.0484 & $\mathbf{2 . 2 4}$ & $\mathbf{9 . 3 3}$ & $\mathbf{0 . 0 2 5 4}$ \\
\hline F2 & 2.40 & 13.87 & 0.0515 & $\mathbf{2 . 3 7}$ & $\mathbf{1 2 . 3 8}$ & $\mathbf{0 . 0 4 0 0}$ \\
\hline F3 & $\mathbf{3 . 1 4}$ & 8.81 & 0.0339 & 4.98 & $\mathbf{6 . 3 5}$ & $\mathbf{0 . 0 2 1 3}$ \\
\hline F4 & $\mathbf{1 . 9 0}$ & 7.71 & 0.0316 & 2.18 & $\mathbf{5 . 3 7}$ & $\mathbf{0 . 0 1 4 1}$ \\
\hline F5 & 2.86 & 9.29 & 0.0306 & $\mathbf{1 . 9 8}$ & $\mathbf{6 . 2 0}$ & $\mathbf{0 . 0 2 0 4}$ \\
\hline F6 & $\mathbf{3 . 9 5}$ & 9.63 & $\mathbf{0 . 0 2 4 2}$ & 4.25 & $\mathbf{8 . 1 0}$ & 0.0263 \\
\hline F7 & $\mathbf{4 . 3 3}$ & 13.72 & 0.0523 & 5.62 & $\mathbf{1 1 . 4 4}$ & $\mathbf{0 . 0 2 3 0}$ \\
\hline F8 & 4.65 & 14.94 & $\mathbf{0 . 0 4 2 7}$ & $\mathbf{2 . 1 1}$ & $\mathbf{8 . 2 6}$ & 0.0433 \\
\hline F9 & 15.40 & 44.56 & 0.0683 & $\mathbf{2 . 6 1}$ & $\mathbf{6 . 7 3}$ & $\mathbf{0 . 0 1 6 8}$ \\
\hline F10 & 13.81 & 54.25 & 0.1017 & $\mathbf{5 . 7 8}$ & $\mathbf{1 2 . 1}$ & $\mathbf{0 . 0 2 6 2}$ \\
\hline F11 & $\mathbf{2 . 3 3}$ & 9.64 & 0.0232 & 3.36 & $\mathbf{4 . 8 5}$ & $\mathbf{0 . 0 1 2 8}$ \\
\hline F12 & $\mathbf{3 . 0 7}$ & $\mathbf{1 2 . 9 8}$ & 0.0482 & 17.02 & 46.74 & $\mathbf{0 . 0 3 2 6}$ \\
\hline F13 & $\mathbf{3 . 4 1}$ & 14.03 & 0.4499 & 4.66 & $\mathbf{1 2 . 7 5}$ & $\mathbf{0 . 0 7 0 3}$ \\
\hline F14 & $\mathbf{2 . 1 8}$ & 15.08 & 0.0763 & 2.56 & 14.74 & $\mathbf{0 . 0 6 8 7}$ \\
\hline F15 & 2.58 & 10.34 & 0.0344 & $\mathbf{2 . 1 6}$ & $\mathbf{4 . 2 5}$ & $\mathbf{0 . 0 1 0 7}$ \\
\hline F16 & $\mathbf{4 . 5 0}$ & $\mathbf{7 . 2 9}$ & 0.0320 & 5.81 & 7.54 & $\mathbf{0 . 0 2 0 8}$ \\
\hline & & & & & \\
\hline
\end{tabular}

a very convenient way to compare the proposed method with other existing approaches (Fillard et al., 2011). The Fiber Cup phantom size is $64 \times 64 \times 3$ voxels with a resolution of $6 \times 6 \times 6 \mathrm{~mm}$. It contains 2 repetitions of 65 gradient directions each, including 1 baseline direction for each repetition. The b-value is 2650 for each direction. Parameters are chosen for each fiber but in most of the cases, 5000 particles were used with a propagation step length of $0.5 \mathrm{~mm}$, a concentration $\kappa=30$ and a resampling threshold $\epsilon_{\mathrm{ESS}}=0.01$.

The quantitative results of our algorithm are summarized in the table 1 and 


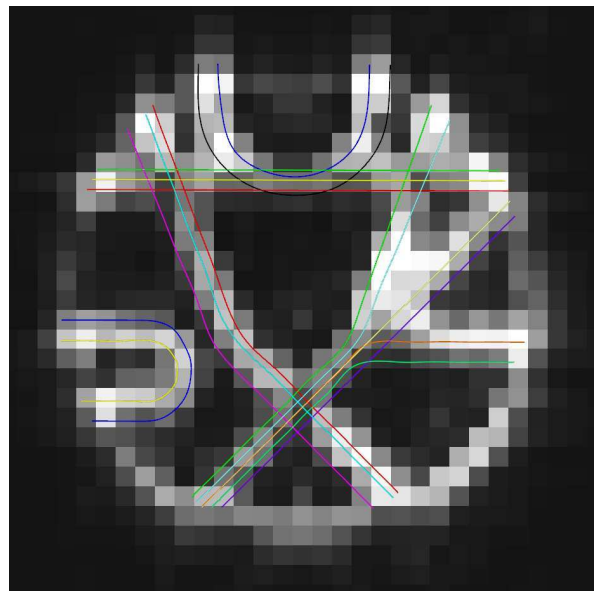

(a) Ground truth

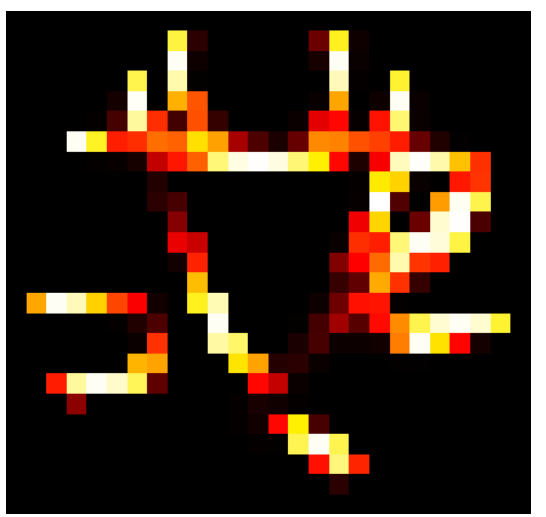

(c) Estimated connectivity map using FSL (Behrens et al., 2007)

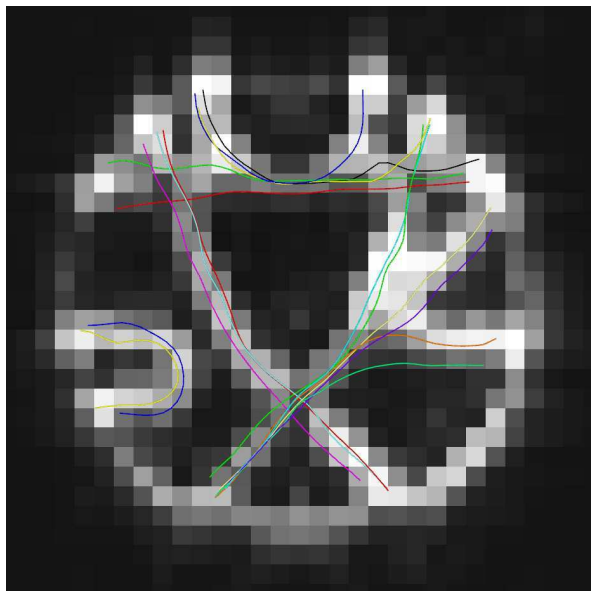

(b) Estimated fiber paths using the proposed algorithm

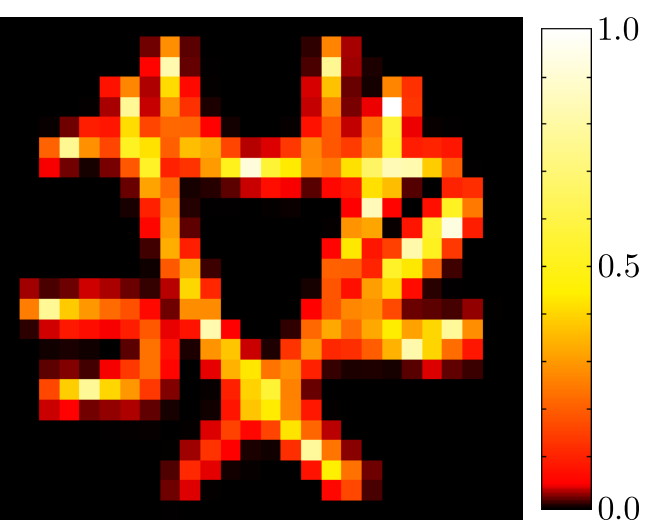

(d) Estimated connectivity map using the proposed algorithm

Figure 6: Fibers trajectories and connectivity map estimations of our algorithm on the Fiber Cup phantom compared respectively to the ground truth and the result of FSL (Behrens et al., 2007). Tractographies of all seeds are displayed on the same map. 
estimated fibers from the particles cloud are shown in figure 6 . The algorithm is also compared to a probabilistic algorithm (Behrens et al., 2003) ${ }^{2}$. The algorithm gets 71 points according to the Fiber Cup contest and would be at the $2^{\text {nd }}$ position in the ranking. Nevertheless, satisfactory results are generated in a short computation time. For instance, the winner of this contest Reisert et al. (2011) uses a global algorithm which performed the tractography in one day of computation time whereas our implementation ran in 2 minutes on a computer with 8 processor Intel Xeon $2.4 \mathrm{GHz}$ (including the preprocessing, i.e. the model estimation, the noise estimation, the maxima extraction, etc.).

\subsection{In-vivo data}

In this section, experiments have been completed on two in-vivo data: adult and fetal brain.

\subsubsection{Adult brain}

Data. First, in-vivo adult brain dataset comes from the MIDAS / National Alliance for Medical Image Community (NAMIC) ${ }^{3}$ and was acquired from a healthy adult volunteer using a 3 Tesla GE system. It contains a $144 \times 144 \times 85$ volume image with $1.7 \times 1.7 \times 1.7 \mathrm{~mm}$ voxel resolution. The diffusion signal was measured in 51 directions with $b=900$ s.mm ${ }^{-2}$ and there are 8 baseline images $\left(b=0 \mathrm{~s} . \mathrm{mm}^{-2}\right)$. During the model estimation, the 8 baseline images have been used by averaging them.

Number of particle parameter. A critical parameter in the proposed methodology is the number of particles used for each seeds. Indeed, the more particles that are used, the more accurate and the slower the algorithm is. Therefore, the accuracy depending on the number of particles is an essential information.

The purpose of this paragraph is to evaluate the convergence of the algorithm. Based on previous experiments, it has been observed that the use of 1000

\footnotetext{
${ }^{2}$ Thanks to Ting-Shuo Yo and Pierre Fillard for their help when setting up FSL's parameters for the phantom of the Fiber Cup contest.

${ }^{3}$ http://insight-journal.org/midas/collection/view/190
} 


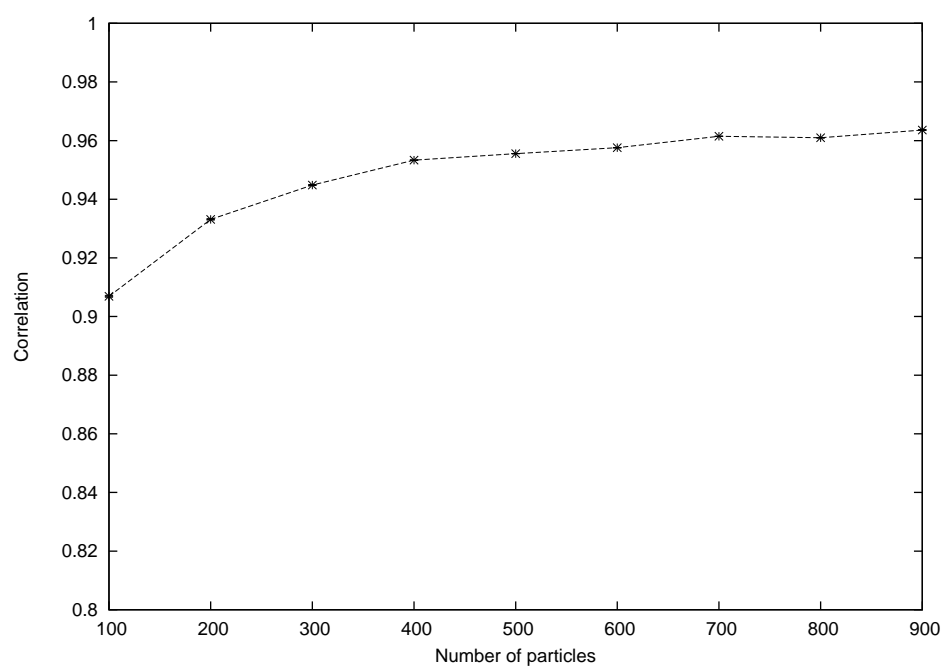

Figure 7: Number of particle parameter study. The similarity measure (correlation) of a tractography of $N$ particles compared to an initial tractography with 1000 particles depending on the number of particles is plotted. This experiment shows how the number of particles parameter influences the accuracy of the solution. The major gain is to decrease the amount of the algorithm run time while preserving a good estimation of the solution searched.

particles provides a good estimation of the posterior density. This experiment consists in estimating probability maps with decreasing numbers of particles and evaluating the quality of the estimates based on a correlation measure with the probability map obtained using 1000 particles. In order to get coherent observations, experiment is made on a large number of seeds (about $10^{5}$ ) in the adult brain data.

Results are plotted in figure 7. The curve seems to stabilize at 400 particles with a correlation of 0.95 . Note that even with 100 particles, the correlation measure is above 0.9. Considering that 1000 particles gives a good estimation, this experiment shows that running the algorithm with a small number of particles (e.g. 100) provides a solution which is a least $90 \%$ correlated to this estimation during shorter elapsed time of computation.

Experiments. The proposed algorithm was applied on adult brain data with a seed spacing of $1 \mathrm{~mm}$, a step length of $0.5 \mathrm{~mm}$, a concentration $\kappa=60$, a resam- 

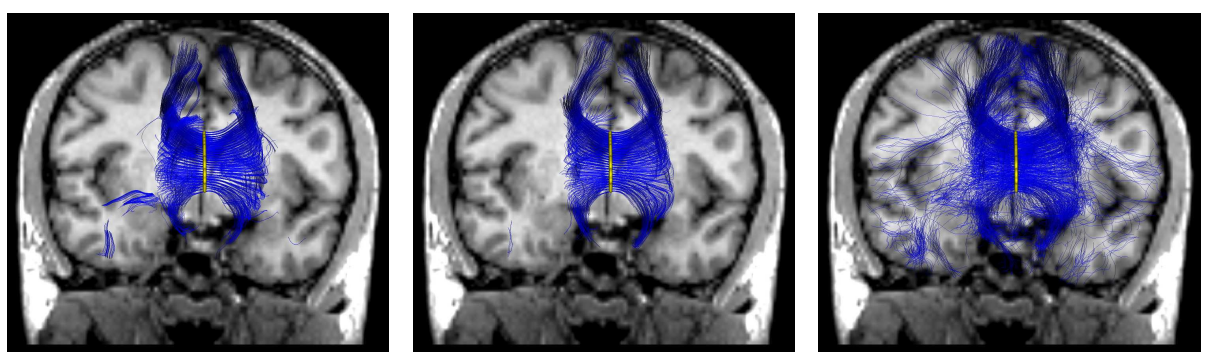

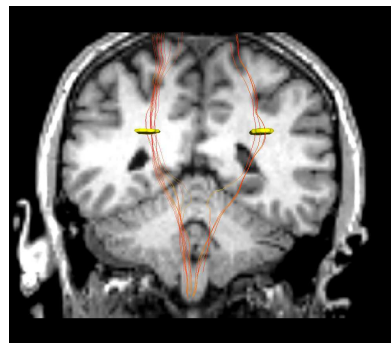

(a) Mori et al. (1999)

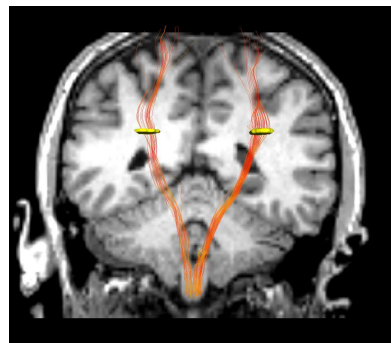

(b) Descoteaux et al. (2009)

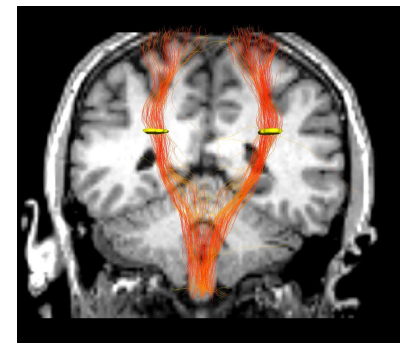

(c) Proposed algorithm

Figure 8: Tractography results of an adult brain using Tensor-based streamline, fODF-based streamline and the proposed algorithm. The first and the last row show respectively tractography of the corpus callosum and the pyramidal fasciculus. The corpus callosum and pyramidal fasciculus are respectively displayed in blue and orange. Seed regions are depicted in yellow.

pling threshold $\epsilon_{\mathrm{ESS}}=5$ and 100 particles. In order to get only motor tracts in the pyramidal fasciculus, the fiber paths estimates were cropped using a region of interest in the brainstem. Comparisons of the proposed algorithm with deterministic streamline algorithms Descoteaux et al. (2009) and Mori et al. (1999) and with probabilistic algorithm Zhang et al. (2009) are respectively displayed in figures 8 and 9-10. The figure 11 illustrate the tractography provided using the proposed method with the full tracts colored by their local orientation.

\subsubsection{Fetal brain}

Data. Second, in-vivo fetal brain dataset was performed on a $1.5 \mathrm{~T}$ Siemens Avanto MRI Scanner (SIEMENS, Erlangen, Germany) using a 6-channel phased array coil combined to the spine array positioned around the mother abdomen. An axial spin echo single-shot echo-planar sequence was acquired in free breath- 


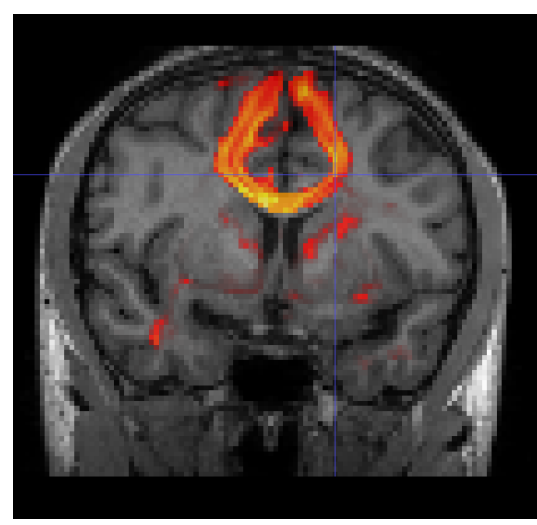

(a) FSL (Behrens et al., 2007)

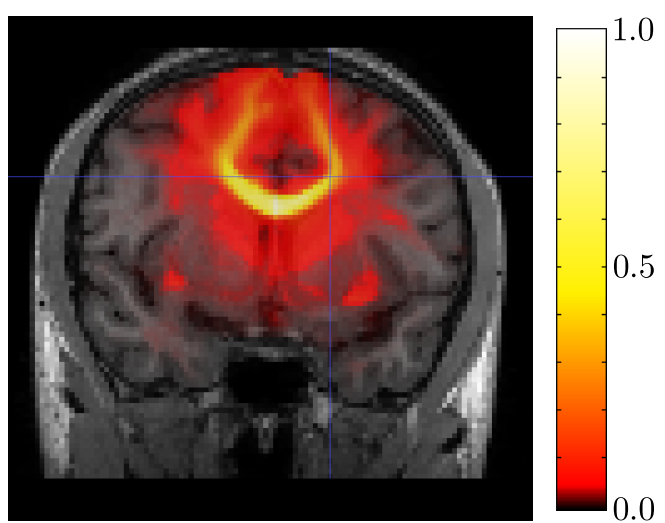

(b) Proposed algorithm

Figure 9: Coronal view of connectivity maps of the corpus callosum tractographies on an adult brain using FSL (Behrens et al., 2007) and the proposed algorithm.

ing along 30 non-collinear diffusion gradient encoding directions with a b value of $700 \mathrm{~s} . \mathrm{mm}^{2}$. The following pulse sequence parameters were used: $\mathrm{TR}=6800 \mathrm{~ms}$; $\mathrm{TE}=99 \mathrm{~ms} ; \mathrm{FOV}=250 \times 250 \mathrm{~mm}^{2} ;$ matrix $=128 \times 128 ; 41$ contiguous axial slices of $3.5 \mathrm{~mm}$ thickness covering the whole fetal brain; no gap; 2 excitations. The resolution of the T2 weighted HASTE sequence (TE/TR $=147 / 3190 \mathrm{~ms})$ is : $0.74 \times 0.74 \times 3.45 \mathrm{~mm}$. The gestation age of the fetus was 32 weeks.

Experiments. The proposed algorithm was applied with a seed spacing of $1 \mathrm{~mm}$, a step length of $0.5 \mathrm{~mm}$, a concentration $\kappa=60$, a resampling threshold $\epsilon_{\mathrm{ESS}}=$ 5 and 100 particles. Comparisons of the proposed algorithm with streamline algorithms Descoteaux et al. (2009) and Mori et al. (1999) and with probabilistic algorithm Zhang et al. (2009) are respectively displayed in figures 12 and 13-14. A whole fetal brain tractography is illustrated in figure 15 .

\subsubsection{Discussion on experiments}

As shows figure 8, tensor-based (Mori et al., 1999) and fODF-based (Descoteaux et al., 2009) streamline algorithms recover close fiber paths solutions with slight local differences. Main structures of corpus callosum and pyramidal tracts are reconstructed. These similar results may be due to the low b-values 

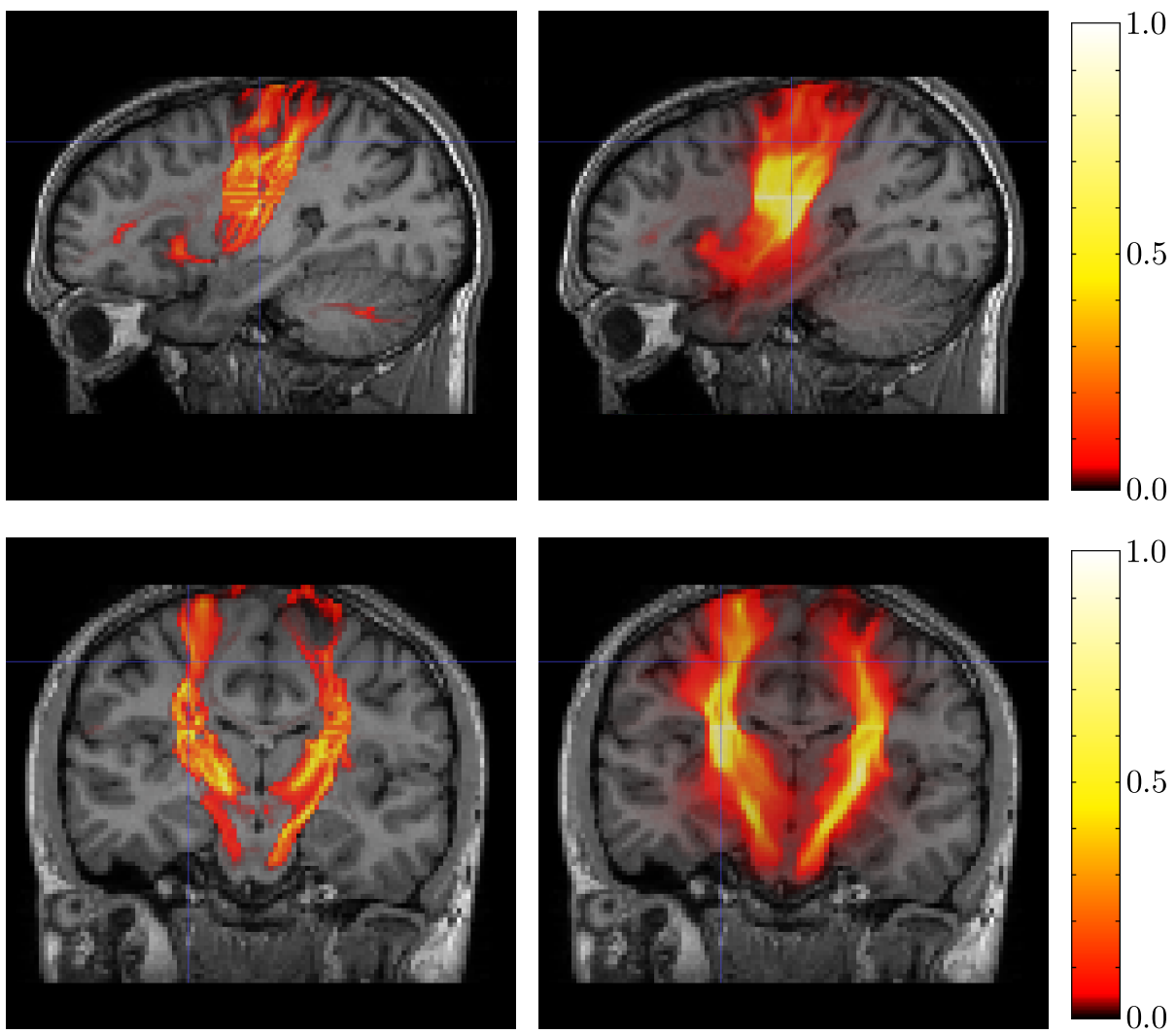

(a) FSL (Behrens et al., 2007)

(b) Proposed algorithm

Figure 10: Connectivity maps of the pyramidal fasciculus tractographies on an adult brain using FSL (Behrens et al., 2007) and the proposed algorithm. Top row: sagittal views ; last row: coronal views. 

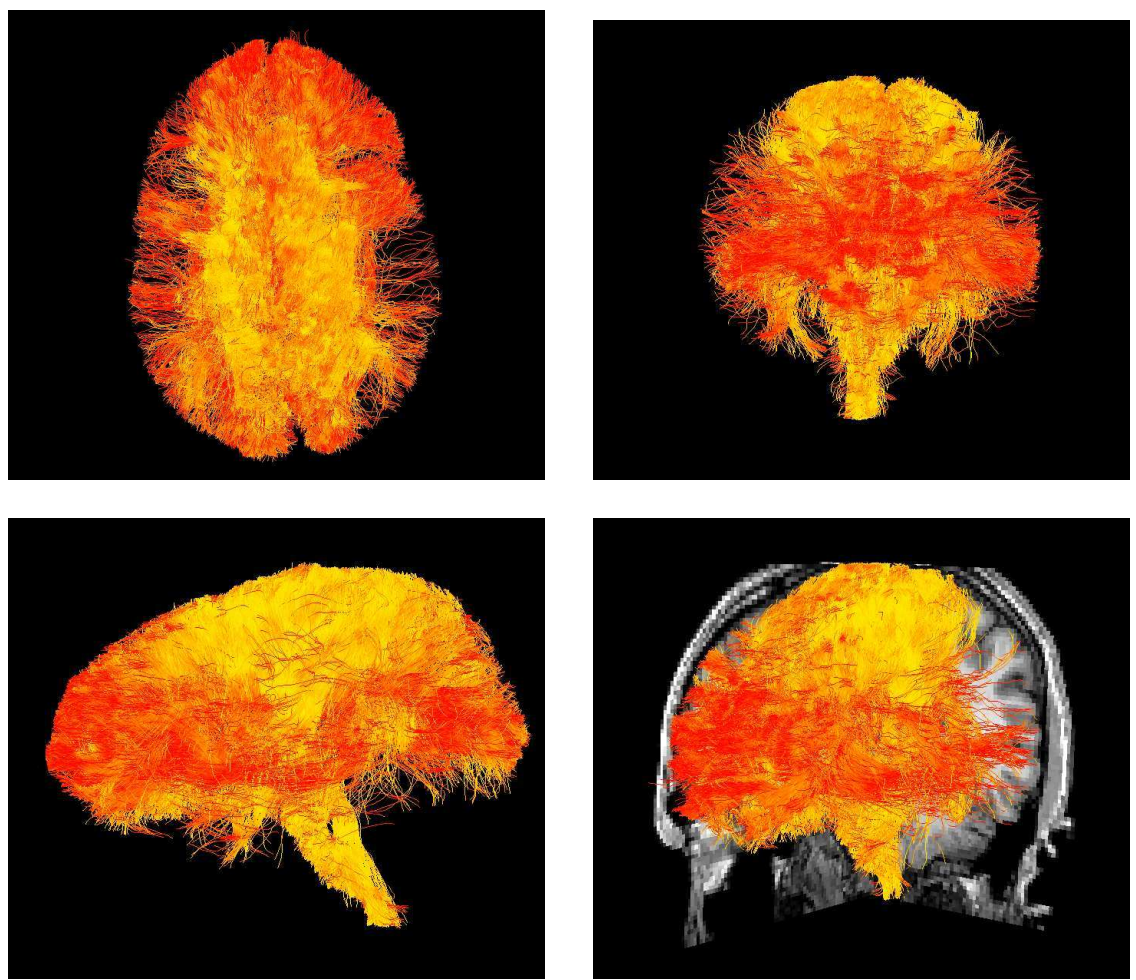

Figure 11: Tractography of the corpus callosum and the pyramidal tracts using the presented algorithm. This is the same result as in figure 8 , but without cutting the pyramidal tracts and with the opacity of the display set to 1 . The tracts are colored by their local orientation (red to yellow for respectively horizontal to vertical axes). 

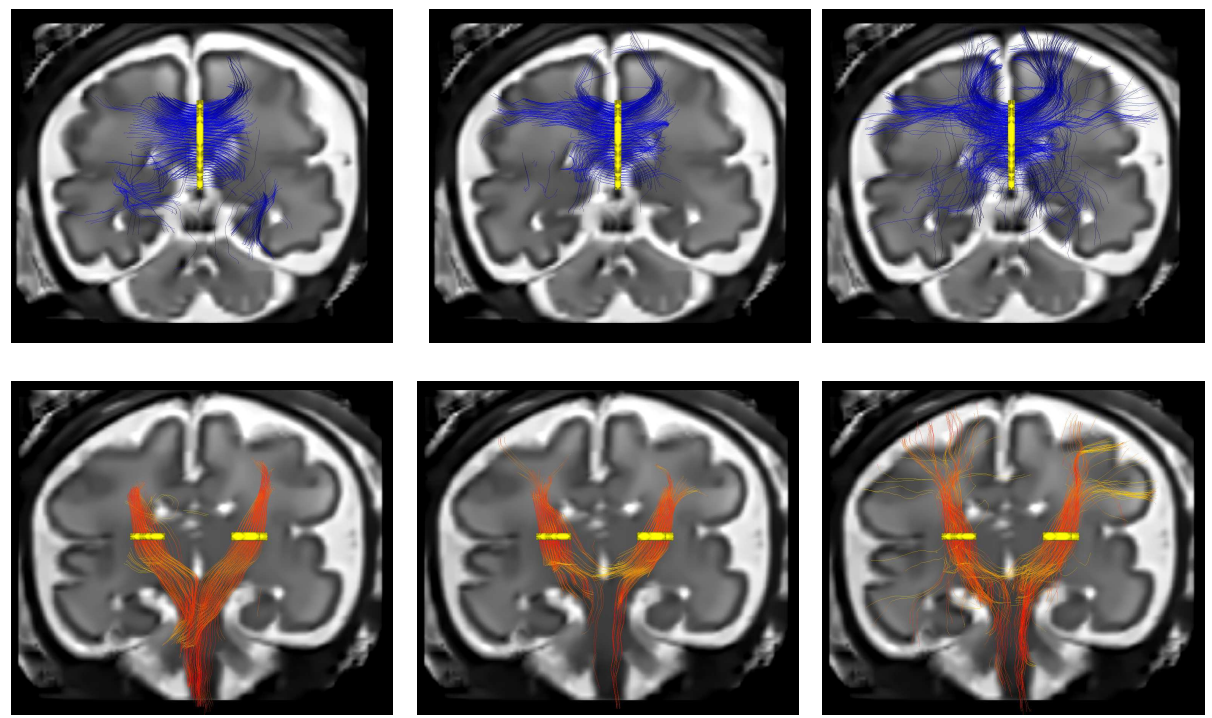

(a) Mori et al. (1999)

(b) Descoteaux et al. (2009)

(c) Proposed algorithm

Figure 12: Tractography results of a fetal brain using Tensor-based streamline, fODF-based streamline and the proposed algorithm. The first and the last row show respectively the sagittal and the coronal views. The corpus callosum and pyramidal fasciculus are respectively displayed in blue and orange. Seed regions are depicted in yellow. The perspective effect of the illustrations suggests that some tracts go out of the brain but this is not the case.

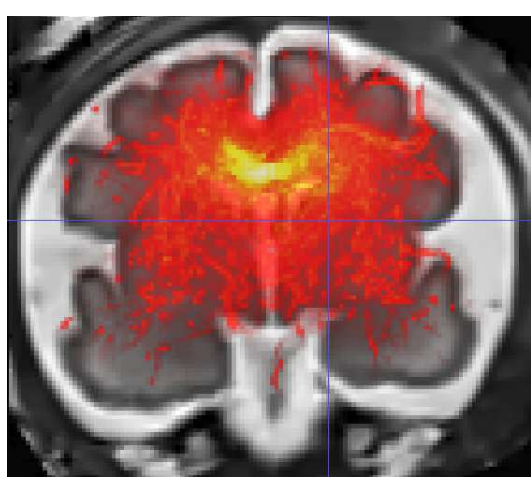

(a) Zhang et al. (2009)

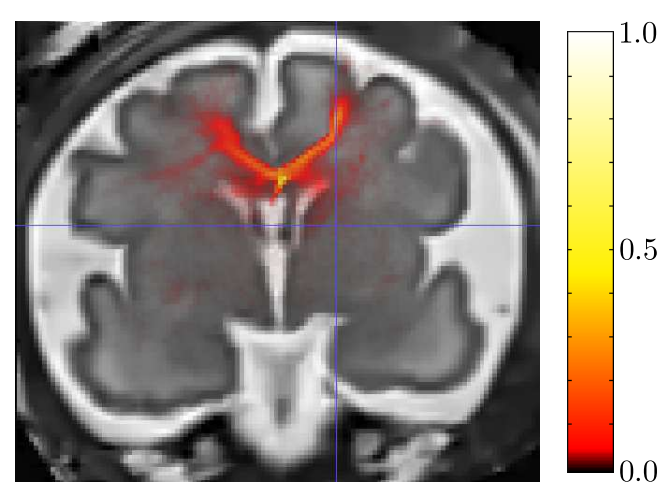

(b) Proposed algorithm

Figure 13: Coronal view of connectivity maps of the corpus callosum tractographies on a fetal brain using our implementation of Zhang et al. (2009) and the proposed algorithm. 

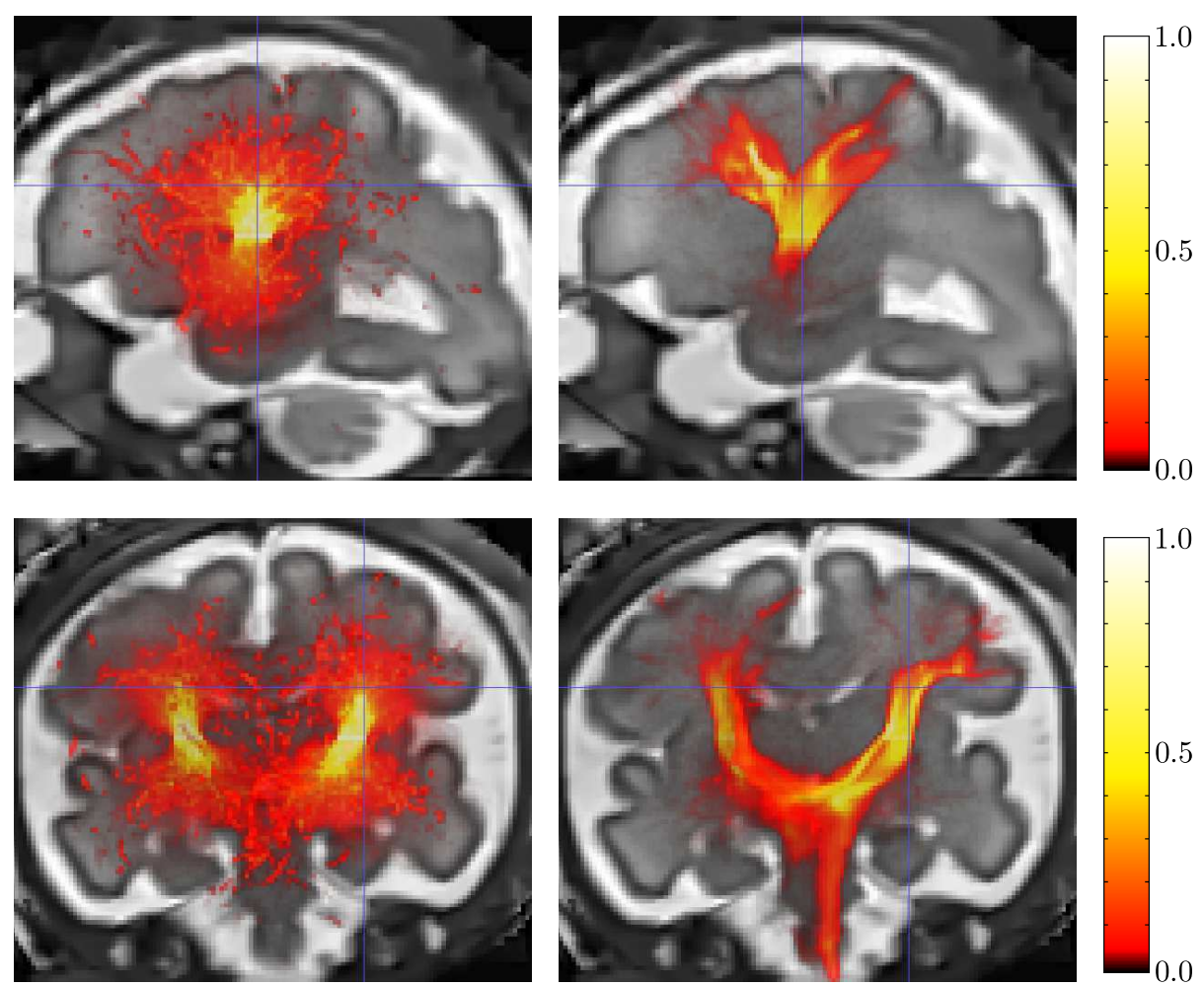

(a) Zhang et al. (2009)

(b) Proposed algorithm

Figure 14: Connectivity maps of the pyramidal fasciculus tractographies on a fetal brain using our implementation of Zhang et al. (2009) and the proposed algorithm. Top row: sagittal views ; last row: coronal views. Because the fetal brain is not perfectly oriented, the coronal view suggests that the solution of the proposed algorithm in the brainstem is not symmetric but this is not the case (see the symmetry of pyramidal tracts of the proposed algorithm in figure 12). 

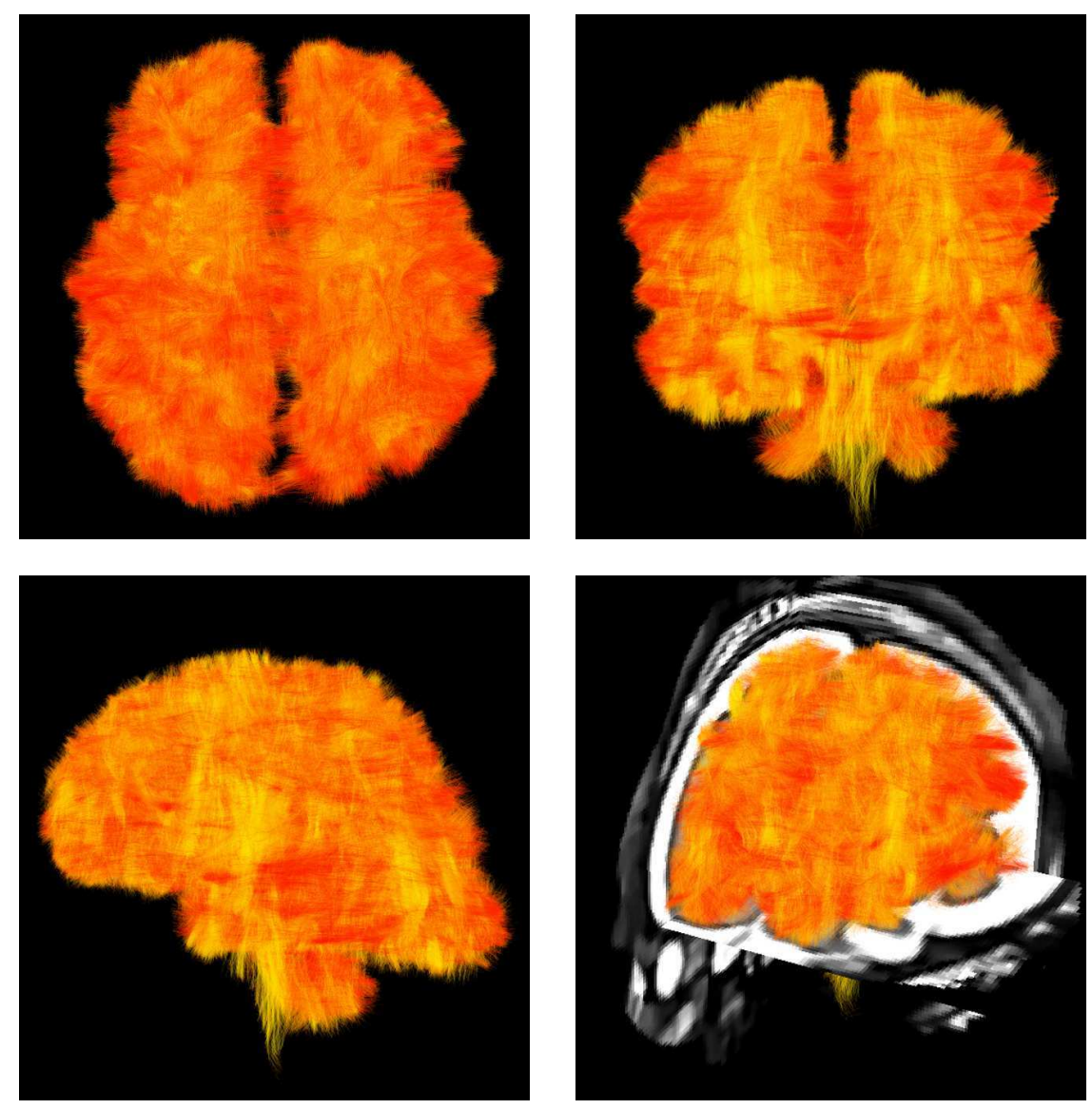

Figure 15: Tractography of the whole fetal brain using the presented tractography technique. The seed region is defined by using a mask of the white matter obtained by tissue segmentation. The seed spacing is $1 \mathrm{~mm}$. The opacity of the display has been set to 0.1 . The tracts are colored by their local orientation (red to yellow for respectively horizontal to vertical axes). 
$(b<1000)$ which tends to make the high order model almost looks like tensor. The proposed method provides long fiber tracts with a small fanning effect at the end (see for instance pyramidal fasciculus and some fibers of the corpus callosum). In fetal brain (see figure 12), tensor-based streamline tractography algorithm produces short tracts. This might be due to low FA in fetal DW-MRI, which prevents the tensor model from providing valuable information on local diffusion configuration. fODF-based algorithm is able to trace longer tracts, allowing it to discern the entire corpus callosum form in coronal view. As in adult brain experiments, the proposed method reveals long fiber paths with a small fanning effect at the end of the tracts (see pyramidal fasciculus and the end of corpus callosum's tracts of the U-shaped structure when entering grey matter). Since some fiber tracts link the two pyramidal fasciculus in both solutions produced by Descoteaux et al. (2009) and the proposed algorithm (figure 12), this might be due to artifacts in Q-Ball model (these two methods use the same diffusion model). The tractographies displayed in figures 11 and 15 illustrate the capacity of the presented algorithm to perform valuable solutions, even with low b-values data.

Results of FSL (Behrens et al., 2007) and of our implementation of Zhang et al. (2009) on in-vivo data (figures 9, 10, 13 and 14) allows to distinguish the searched structures (corpus callosum and pyramidal fasciculus) with having a visualization of uncertainty. The proposed method offers in the presented experiments an accurate description of the searched structures (see for instance the sagittal views of adult and fetal pyramidal tracts in figure 10 and 14) and is able to recover pyramidal fasciculus in the brainstem in both experiments. Fetal connectivity maps (figures 13 and 14) seems to be smoother and to have more uncertainty than adult connectivity maps. Nevertheless, the desired fiber bundles (corpus callosum and pyramidal fasciculus) are still recognizable. 


\section{Discussion}

In this article, a probabilistic tractography method has been presented based on the well-formalized particle filtering framework and using a high order model for diffusion data. The proposed method is not model-dependent: any model providing ODF can be used. The output of the algorithm is an estimate of the posterior density of the white matter fibers. The keypoints of the method are the three densities: prior, likelihood, importance. First, the prior density constrains the solution. Then, the likelihood density ensures the reasonableness of the estimation with a noise model of DW-MRI data. Finally, fast and efficient sampling is realized by a vMF mixture.

The proposed algorithm is an improvement of the method described in Zhang et al. (2009). The basic idea of particle filtering tracking process is kept but densities have been adapted to high order model requirements using spherical diffusion function, such as Q-Ball models. For instance, the use of a vMF mixture as importance density allows to use all the information provided by the ODF. Using an optimal algorithm for MAP estimation (Godsill et al., 2001) instead of considering particles' importance weights (Zhang et al., 2009), the MAP is better estimated and therefore the fiber's trajectory estimation is more accurate.

As shown by the experiments performed on the synthetic dataset and the Fiber Cup phantom, the use of a Q-ball data modeling within the particle filtering framework leads to accurate estimations of complex fiber configurations. Experiments on in vivo data have shown the contribution of the particle filtering framework compared to deterministic streamline approaches and tensor-based particle filtering algorithm. Using the proposed method, main structures are recovered, fiber tracts end with a fanning effect and connectivity maps describe accurately the main structures and uncertainty.

Although the proposed method shows interesting results on the Fiber Cup phantom (see section 3.2), the algorithm produces a small wavering effect on the fiber estimates. This loss of precision is mainly due to the chosen ODF model, 
which is more sensitive to noise than the tensor model (tensors are smooth modeling of diffusion signal). A stronger global regularization than concentration parameter of local vMF density or a more recent diffusion model could provide smoother estimates. Using another dynamic model (see equation 1) is also a way to have a stronger constraint on the estimates. Furthermore, optimal tractography results are obtained on the Fiber Cup phantom by setting slightly different parameters. These parameters add flexibility to the proposed method which is able to capture many different connection configurations, as shown by the Fiber Cup phantom.

Initially, the particle filtering framework is not fast enough for whole brain tractography applications in a short computation time. It is more suited for local tractography of specific fiber bundles. However, as established in section 3.3.1, the number of particle parameter has a major effect on algorithm's speed and can be decreased without having a substantial negative impact on the estimated solution. Therefore, the whole brain tractography is practicable for reasonable computation time. For instance, the computation time of a particle filter propagation from one seed in an adult brain is about $40 \mathrm{~s}$ using 100 particles and $3 \mathrm{mn}$ using 1000 particles on a computer with one processor Intel Core 2 Duo $2.93 \mathrm{GHz}$ (including the preprocessing, i.e. the model estimation, the noise estimation, the maxima extraction, etc.).

Further work could study the whole diffusion information available, without any diffusion model constraint. Indeed, techniques for estimating models induce approximations that can exaggerate uncertainty in the data. Other improvement could involve parameters of the tracking process. For instance, the step size $\lambda$ (cf. section 2.2) can be estimated by the full 3D ensemble average propagator (Descoteaux et al., 2011), which gives radial and angular information. The use of recent spherical constrained deconvolution models (Aganj et al., 2010, Tournier et al., 2007) could improve the capture of low angle crossing configurations at high b-values. Furthermore, it would be interesting to define the likelihood in an analytical formula depending on direction parameters to 
improve speed and accuracy of the proposed algorithm.

Acknowledgment: The research leading to these results has received funding from the European Research Council under the European Community's Seventh Framework Programme (FP7/2007-2013 Grant Agreement no. 207667). This work is also funded by NIH Grant R01 NS055064.

\section{Appendix A. Diffusion modeling}

\section{Appendix A.1. Spherical harmonics}

Spherical harmonics are the angular portion of a set of solutions to Laplace's equation. They are generally used for representing spherical functions, such as the diffusion signal. Given spherical coordinates $(\theta, \phi) \in[0, \pi] \times[0,2 \pi[$, the spherical harmonic basis at order $l$ and degree $m$ is written as

$$
\mathrm{Y}_{l}^{m}(\theta, \phi)=\sqrt{\frac{2 l+1}{4 \pi} \frac{(l-m) !}{(l+m) !}} \mathrm{P}_{l}^{m}(\cos \theta) \mathrm{e}^{i m \phi},
$$

where $\mathrm{P}_{l}^{m}$ is the associated Legendre function. Since the assumption of the diffusion signal being real and antipodally symmetric is made, the use of real spherical harmonics is more suitable:

$$
\mathcal{Y}_{l}^{m}(\theta, \phi)=\left\{\begin{array}{ll}
(-1)^{m} \sqrt{2} \mathcal{R} e\left(\mathrm{Y}_{l}^{m}(\theta, \phi)\right) & \text { if }-l \leq m<0 \\
\mathrm{Y}_{l}^{0} & \text { if } m=0 \\
\sqrt{2} \mathcal{I} m\left(\mathrm{Y}_{l}^{m}(\theta, \phi)\right) & \text { if } 0<m \leq l
\end{array} .\right.
$$

Appendix A.2. Diffusion signal

Let us consider an image domain $\Omega \subset \mathbb{R}^{3}$ and diffusion weighted measurement in $N$ directions. According to the spherical harmonics decomposition model (Descoteaux et al., 2007), the diffusion signal at a voxel $x \in \Omega$ and in each of the $N$ directions $u=(\theta, \phi)$ is decomposed into real spherical harmonics (A.2):

$$
S_{x}(u)=\sum_{k=0}^{l} \sum_{m=-k}^{k} c_{k}^{m} \mathcal{Y}_{k}^{m}(u)
$$


where $l$ is the order of the decomposition and only even orders are used. The coefficients $c_{k}^{m}$ can be estimated by a regularized linear regression (Descoteaux et al., 2006, 2007).

\section{Appendix A.3. Fiber orientation distribution function (fODF)}

Applying the Funk-Radon transform to (A.3), the diffusion ODF can be analytically computed at point $x \in \Omega$ and in direction $u=(\theta, \phi)$ (Descoteaux et al., 2007). In order to get a sharper ODF, the fiber orientation distribution function (fODF) is computed from the diffusion ODF by a deconvolution (Descoteaux et al., 2009). Using coefficients estimated from equation (A.3), this function is defined at a point $x \in \Omega$ and in a direction $u=(\theta, \phi)$ as

$$
\psi_{x}(u)=\sum_{k=0}^{l} \sum_{m=-k}^{k} 2 \pi \mathrm{P}_{k}^{m}(0) \frac{c_{k}^{m}}{f_{k}^{m}} \mathcal{Y}_{k}^{m}(u)
$$

where $P_{k}^{m}$ is the associated Legendre function, $c_{k}^{m}$ are the estimated coefficient in (A.3) and $f_{k}^{m}$ are the sharper coefficients (see Descoteaux et al. (2009) for more details).

\section{References}

Aganj, I., Lenglet, C., Sapiro, G., 2010. Odf maxima extraction in spherical harmonic representation via analytical search space reduction. In: Jiang, T., Navab, N., Pluim, J., Viergever, M. (Eds.), Medical Image Computing and Computer-Assisted Intervention MICCAI 2010. Vol. 6362 of Lecture Notes in Computer Science. Springer Berlin / Heidelberg, pp. 84-91.

Barmpoutis, A., Jian, B., Vemuri, B., 2009. Adaptive kernels for multi-fiber reconstruction. In: Prince, J., Pham, D., Myers, K. (Eds.), Information Processing in Medical Imaging. Vol. 5636 of Lecture Notes in Computer Science. Springer Berlin / Heidelberg, pp. 338-349.

Barnett, A., 2009. Theory of q-ball imaging redux: Implications for fiber tracking. Magnetic Resonance in Medicine 62 (4), 910-923. 
Basser, P., Mattiello, J., LeBihan, D., Jan. 1994. MR diffusion tensor spectroscopy and imaging. Biophysical Journal 66 (1), 259-267.

Basser, P. J., Pajevic, S., Pierpaoli, C., Duda, J., Aldroubi, A., 2000. In vivo fiber tractography using DT-MRI data. Magnetic Resonance in Medicine 44 (4), 625-632.

Behrens, T., Berg, H. J., Jbabdi, S., Rushworth, M., Woolrich, M., Jan. 2007. Probabilistic diffusion tractography with multiple fibre orientations: What can we gain? NeuroImage 34 (1), 144-155.

Behrens, T., Woolrich, M., Jenkinson, M., Johansen-Berg, H., Nunes, R., Clare, S., Matthews, P., Brady, J., Smith, S., 2003. Characterization and propagation of uncertainty in diffusion-weighted MR imaging. Magnetic Resonance in Medicine 50 (5), 1077-1088.

Berman, J. I., Chung, S., Mukherjee, P., Hess, C. P., Han, E. T., Henry, R. G., 2008. Probabilistic streamline q-ball tractography using the residual bootstrap. NeuroImage 39 (1), $215-222$.

Bjornemo, M., Brun, A., Kikinis, R., Westin, C., 2002. Regularized stochastic white matter tractography using diffusion tensor MRI. In: Dohi, T., Kikinis, R. (Eds.), Medical Image Computing and Computer-Assisted Intervention MICCAI 2002. Vol. 2488 of Lecture Notes in Computer Science. Springer Berlin / Heidelberg, pp. 435-442.

Bloy, L., Verma, R., 2008. On computing the underlying fiber directions from the diffusion orientation distribution function. In: Medical Image Computing and Computer-Assisted Intervention-MICCAI 2008. pp. 1-8.

Descoteaux, M., Angelino, E., Fitzgibbons, S., Deriche, R., 2006. Apparent diffusion coefficients from high angular resolution diffusion imaging: Estimation and applications. Magnetic Resonance in Medicine 56 (2), 395-410. 
Descoteaux, M., Angelino, E., Fitzgibbons, S., Deriche, R., 2007. Regularized, fast, and robust analytical q-ball imaging. Magnetic Resonance in Medicine $58(3), 497-510$.

Descoteaux, M., Deriche, R., Bihan, D. L., Mangin, J., Poupon, C., Aug. 2011. Multiple q-shell diffusion propagator imaging. Medical Image Analysis 15 (4), 603-621.

Descoteaux, M., Deriche, R., Knosche, T., Anwander, A., 2009. Deterministic and probabilistic tractography based on complex fibre orientation distributions. Medical Imaging, IEEE Transactions on 28 (2), 269-286.

Doucet, A., Godsill, S., Andrieu, C., 2000. On sequential monte carlo sampling methods for bayesian filtering. Statistics and computing 10 (3), 197-208.

Fillard, P., Descoteaux, M., Goh, A., Gouttard, S., Jeurissen, B., Malcolm, J., Ramirez-Manzanares, A., Reisert, M., Sakaie, K., Tensaouti, F., Yo, T., Mangin, J., Poupon, C., May 2011. Quantitative evaluation of 10 tractography algorithms on a realistic diffusion MR phantom. NeuroImage 56 (1), 220-234.

Fillard, P., Poupon, C., Mangin, J., 2009. A novel global tractography algorithm based on an adaptive spin glass model. In: Yang, G., Hawkes, D., Rueckert, D., Noble, A., Taylor, C. (Eds.), Medical Image Computing and ComputerAssisted Intervention - MICCAI 2009. Vol. 5761 of Lecture Notes in Computer Science. Springer Berlin / Heidelberg, pp. 927-934.

Friman, O., Farneback, G., Westin, C., 2006. A bayesian approach for stochastic white matter tractography. Medical Imaging, IEEE Transactions on 25 (8), 965-978.

Gasser, T., Sroka, L., Jennen-Steinmetz, C., 1986. Residual variance and residual pattern in nonlinear regression. Biometrika 73 (3), 625-633.

Godsill, S., Doucet, A., West, M., 2001. Maximum a posteriori sequence estimation using monte carlo particle filters. Annals of the Institute of Statistical Mathematics 53 (1), 82-96. 
Jbabdi, S., Woolrich, M., Andersson, J., Behrens, T., 2007. A bayesian framework for global tractography. NeuroImage 37 (1), 116 - 129.

Jeurissen, B., Leemans, A., Jones, D. K., Tournier, J., Sijbers, J., 2011. Probabilistic fiber tracking using the residual bootstrap with constrained spherical deconvolution. Human Brain Mapping 32 (3), 461-479.

Jiao, F., Gur, Y., Johnson, C., Joshi, S., 2011. Detection of crossing white matter fibers with high-order tensors and rank-\&lt;i\&gt;k\&lt;/i\&gt; decompositions. In: Szkely, G., Hahn, H. (Eds.), Information Processing in Medical Imaging. Vol. 6801 of Lecture Notes in Computer Science. Springer Berlin / Heidelberg, pp. 538-549.

Kong, A., Liu, J. S., Wong, W. H., 1994. Sequential imputations and bayesian missing data problems. Journal of the American Statistical Association 89 (425), 278-288.

Lazar, M., 2010. Mapping brain anatomical connectivity using white matter tractography. NMR in Biomedicine 23 (7), 821-835.

Lazar, M., Alexander, A. L., Jan. 2005. Bootstrap white matter tractography (BOOT-TRAC). NeuroImage 24 (2), 524-532.

Lenglet, C., Campbell, J., Descoteaux, M., Haro, G., Savadjiev, P., Wassermann, D., Anwander, A., Deriche, R., Pike, G., Sapiro, G., Siddiqi, K., Thompson, P., 2009. Mathematical methods for diffusion MRI processing. NeuroImage 45 (1, Supplement 1), S111 - S122, mathematics in Brain Imaging.

Lifshits, S., Tamir, A., Assaf, Y., 2009. Combinatorial fiber-tracking of the human brain. NeuroImage 48 (3), $532-540$.

Liu, C., Bammer, R., Acar, B., Moseley, M. E., 2004. Characterizing nongaussian diffusion by using generalized diffusion tensors. Magnetic Resonance in Medicine 51 (5), 924-937. 
Liu, J. S., 1996. Metropolized independent sampling with comparisons to rejection sampling and importance sampling. Statistics and Computing 6 (2), $113-119$.

Malcolm, J. G., Michailovich, O., Bouix, S., Westin, C., Shenton, M. E., Rathi, Y., 2010. A filtered approach to neural tractography using the watson directional function. Medical Image Analysis 14 (1), 58-69.

Mori, S., Crain, B. J., Chacko, V. P., Zijl, P. C. M. V., 1999. Three-dimensional tracking of axonal projections in the brain by magnetic resonance imaging. Annals of Neurology 45 (2), 265-269.

Nowak, R., 1999. Wavelet-based rician noise removal for magnetic resonance imaging. Image Processing, IEEE Transactions on 8 (10), 1408-1419.

Özarslan, E., Mareci, T. H., 2003. Generalized diffusion tensor imaging and analytical relationships between diffusion tensor imaging and high angular resolution diffusion imaging. Magnetic Resonance in Medicine 50 (5), 955965.

Parker, G., Alexander, D., 2003. Probabilistic monte carlo based mapping of cerebral connections utilising whole-brain crossing fibre information. In: Information Processing in Medical Imaging. pp. 684-695.

Parker, G., Wheeler-Kingshott, C., Barker, G., 2002. Estimating distributed anatomical connectivity using fast marching methods and diffusion tensor imaging. Medical Imaging, IEEE Transactions on 21 (5), 505-512.

Poupon, C., Rieul, B., Kezele, I., Perrin, M., Poupon, F., Mangin, J., 2008. New diffusion phantoms dedicated to the study and validation of highangular-resolution diffusion imaging (HARDI) models. Magnetic Resonance in Medicine 60 (6), 1276-1283.

Reisert, M., Mader, I., Anastasopoulos, C., Weigel, M., Schnell, S., Kiselev, V., Jan. 2011. Global fiber reconstruction becomes practical. NeuroImage 54 (2), 955-962. 
Savadjiev, P., Rathi, Y., Malcolm, J., Shenton, M., Westin, C. F., 2010. A Geometry-Based particle filtering approach to white matter tractography. In: Jiang, T., Navab, N., Pluim, J., Viergever, M. (Eds.), Medical Image Computing and Computer-Assisted Intervention - MICCAI 2010. Vol. 6362 of Lecture Notes in Computer Science. Springer Berlin / Heidelberg, pp. 233-240.

Schultz, T., Seidel, H.-P., nov.-dec. 2008. Estimating crossing fibers: A tensor decomposition approach. Visualization and Computer Graphics, IEEE Transactions on 14 (6), $1635-1642$.

Staempfli, P., Jaermann, T., Crelier, G., Kollias, S., Valavanis, A., Boesiger, P., Mar. 2006. Resolving fiber crossing using advanced fast marching tractography based on diffusion tensor imaging. NeuroImage 30 (1), 110-120.

Tournier, J.-D., Calamante, F., Connelly, A., 2007. Robust determination of the fibre orientation distribution in diffusion mri: Non-negativity constrained super-resolved spherical deconvolution. NeuroImage 35 (4), 1459 - 1472.

Tristan-Vega, A., Westin, C.-F., Aja-Fernndez, S., 2009. Estimation of fiber orientation probability density functions in high angular resolution diffusion imaging. NeuroImage 47 (2), $638-650$.

Tuch, D. S., 2004. Q-ball imaging. Magnetic Resonance in Medicine 52 (6), 1358-1372.

Ulrich, G., 1984. Computer generation of distributions on the m-Sphere. Journal of the Royal Statistical Society. Series C (Applied Statistics) 33 (2), 158-163.

Wu, X., Xu, Q., Xu, L., Zhou, J., Anderson, A. W., Ding, Z., 2009. Genetic white matter fiber tractography with global optimization. Journal of Neuroscience Methods $184(2), 375$ - 379 .

Zhang, F., Hancock, E. R., Goodlett, C., Gerig, G., 2009. Probabilistic white matter fiber tracking using particle filtering and von Mises-Fisher sampling. Medical Image Analysis 13 (1), 5-18. 